Japan. J. Math.

Vol. 10, No. 2, 1984

\title{
Nonlinear evolution operators in a Fréchet space
}

\author{
Dedicated to Professor Haruo Sunouchi on his sixtieth birthday
}

\author{
By Kazuo Kobayasi and Shinnosuke OHaru
}

(Received October 15, 1983)

This paper is concerned with nonlinear evolution operators associated with systems of time-dependent evolution equations of the form $u^{\prime}(t) \in A(t) u(t)$, $s<t<T$, in a Fréchet space $X$, where $0 \leqq s<T$ and $\{A(t): 0 \leqq t \leqq T\}$ is a family of nonlinear (possibly multi-valued) operators in $X$. A notion of weak solution to the evolution equation as mentioned above is introduced and the construction of evolution operators providing the weak solutions is discussed.

\section{Introduction}

Let $X$ be a real Fréchet space and consider the system of nonlinear evolution equations in $X$

$$
u^{\prime}(t) \in A(t) u(t), \quad s<t<T,
$$

with initial conditions

$$
u(s)=v,
$$

where $0 \leqq s<T, u$ stands for an $X$-valued unknown function on the interval $[s, T]$ and $\{A(t): t \in[0, T]\}$ is a given family of nonlinear (possibly multivalued) operators in $X$.

Suppose for the moment that there is a subset $X_{0}$ of $X$, and that for every $s \in[0, T]$ and every $v \in X_{0}$, the initial-valued problem for $(D E)_{s}$ with $(I C)_{s}$ has a unique exact solution $u(\cdot, s ; v)$ on $[s, T]$ such that $u(t, s ; v) \in X_{0}$ for $s \leqq t \leqq T$. Then, given a pair $s, t \in[0, T]$ with $s \leqq t$, the operator $U(t, s)$ in $X$ defined by

$$
U(t, s) v=u(t, s ; v) \text { for } v \in X_{0} \text { and } t \in[s, T]
$$

maps $X_{0}$ into itself and is expected to have the following properties:

$\left(\mathbf{E}_{1}\right)$ For $r, s, t \in[0, T]$ with $r \leqq s \leqq t, U(s, s)$ is the identity operator $I$ on $X_{0}$ and $U(t, s) U(s, r)=U(t, r)$ on $X_{0}$.

This work is partly supported by Grant-in-Aid for Scientific Research from the Ministry of Education of Japan. 
$\left(\mathbf{E}_{2}\right)$ For $v \in X_{0}$, the $X$-valued function $U(t, s) v$ is strongly continuous over the triangle $0 \leqq s \leqq t \leqq T$ with respect to $(s, t)$.

Moreover, if the solution $u(\cdot, s ; v)$ depends continuously upon initial-data $v \in X_{0}$, one will have that each $U(t, s)$ is continuous on $X_{0}$. In this paper a family $\mathscr{U}$ of continuous operators $U(t, s)$ from $X_{0}$ into itself with the properties $\left(\mathbb{E}_{1}\right)$ and $\left(\mathbf{E}_{2}\right)$ will be called an evolution operator on $X_{0}$.

We are mainly concerned with evolution equations in a nonreflexive Fréchet space. Hence equations $(D E)_{s}, s \in[0, T)$, do not necessarily have exact solutions even if the $t$-dependence of $A(t)$ is smooth and no matter how initial data are chosen in $X_{0}$. In this paper we employ the following type of generalized solutions to $(D E)_{s}$ : Let $C$ be a closed subset of $X$ and let $s \in$ $[0, T)$. An $X$-valued, strongly continuous function $u(\cdot)$ on $[s, T]$ is said to be a weak solution of $(D E)_{s}$ constrained in $C$, if there exists a sequence $\left(u_{n}(\cdot)\right)$ in $\mathscr{C}([0, T] ; X)$ with the following properties:

$\left(\mathbf{w}_{1}\right)$ For each $n \geqq 1$, there exists a partition

$$
\Delta_{n}=\left\{s=t_{0}^{n}<t_{1}^{n}<\cdots<t_{N(n)}^{n}=T\right\}
$$

of $[s, T]$ and an $X$-valued step function

$$
f_{n}(t)=\sum_{k=1}^{N(n)} z_{k}^{n} \chi_{\left(t_{k-1}^{n}, t_{k}^{n}\right]}(t)
$$

such that for each $k \in\{1,2, \cdots, N(n)\}, u_{n}\left(t_{k}^{n}\right) \in D\left(A\left(t_{k}^{n}\right)\right) \cap C$ and

$$
\frac{u_{n}\left(t_{k}^{n}\right)-u_{n}\left(t_{k-1}^{n}\right)}{t_{k}^{n}-t_{k-1}^{n}}-f_{n}\left(t_{k}^{n}\right) \in A\left(t_{k}^{n}\right) u\left(t_{k}^{n}\right) .
$$

$\left(\mathbf{w}_{2}\right) \quad\left|\Delta_{n}\right| \equiv \max _{k}\left|t_{k}^{n}-t_{k-1}^{n}\right|$ tends to 0 and $f_{n}(t)$ converges in $X$ to 0 as $n \rightarrow$ $\infty$, uniformly for $t \in[s, T]$.

$\left(\mathbf{w}_{3}\right) u_{n}(t)$ converges in $X$ to $u(t)$ as $n \rightarrow \infty$, uniformly for $t \in[s, T]$. (Hence the range of $u(\cdot)$ is contained in the closed set $C$.)

Our objective here is to make an attempt to develop a generation theory for nonlinear evolution operators in $X$ which provide weak solutions to the system $\left\{(D E)_{s}\right\}$ as mentioned above. We discuss the generation of evolution operators on a suitable subspace of the Fréchet space $X$ which can be embedded into a Banach space in a local sense. The method we employ is based on what was developed in the authors' recent work [7]. Moreover, in Section 6 , we shall treat the application of our results to quasilinear hyperbolic equations.

\section{$\S 1$. Preliminaries}

Let $X$ be a real Fréchet space. By an operator $A$ in $X$ we mean a 
(possibly multi-valued) operator with domain $D(A)$ and range $R(A)$ in $X$, where $D(A)=\{u \in X: A u \neq \phi\}$ and $R(A)=\cup\{A u: u \in D(A)\}$. In what follows operators in $X$ are identified with their graphs in $X \times X$; hence we sometimes write $(u, v) \in A$ when $v \in A u$. If $A u$ is a singleton set $\{v\}$ for each $u \in D(A)$, we write $v=A u$ and say that $A$ is a single-valued operator. The restriction of $A$ to a set $C$ is denoted by $A_{C}$. We often treat elements in the range of an operator of the form $I-\lambda A_{c}$, where $I$ is the identity operator. We sometimes write $w=u-\lambda v,(u, v) \in A, u \in C$ if we have the relation $w \in\left(I-\lambda A_{C}\right) u$. Suppose that $I-\lambda A_{C}$ is injective for $\lambda$ in an interval $\left(0, \lambda_{0}\right)$. Then the operator-valued functions

$$
J_{C}(\lambda)=\left(I-\lambda A_{C}\right)^{-1}, \quad A_{c}(\lambda)=\lambda^{-1}\left[J_{c}(\lambda)-I\right]
$$

are called the resolvent of $A_{c}$ and the Yosida approximation of $A_{c}$, respectively.

We think of proper lower semicontinuous nonnegative and convex functionals to classify the class of initial-data and to specify the $t$-dependence of the operator $A(t)$, the stability of solutions of $(D E)_{s}$, etc.. We permit ourselves the common abbreviation, an l.s.c, functional on $X$, in referring to a lower semicontinuous functional on $X$. Let $\mathfrak{p}: X \rightarrow[0, \infty]$ be an l.s.c. seminorm on $X$; hence the effective domain $D(\mathfrak{p}) \equiv\{v \in X ; \mathfrak{p}(v)<\infty\}$ is a linear manifold in $X$. Using the seminorm $\mathfrak{p}$ we define a family $\left\{X_{\alpha}: \alpha>0\right\}$ of subsets of $X$ and a set $X_{0}$ by

$$
X_{\alpha}=\{v \in X: \mathfrak{p}(v) \leqq \alpha\}, \quad \alpha>0, \quad \text { and } X_{0}=\left\{X_{\alpha}: \alpha>0\right\},
$$

respectively. We note that each $X_{\alpha}$ is a closed and absolutely convex subset of $X$, and that $D(p)=X_{0}$.

In order to specify the $t$-dependence of $A(t)$, we introduce two classes of functions on $[0, T] \times[0, T]$. A nonnegative, bounded measurable function $\theta$ defined on all of $[0, T] \times[0, T]$ is said to belong to class $\mathscr{C}$ if it satisfies the following two conditions:

(i) For each $s \in[0, T], \theta(\cdot, s)$ is upper semicontinuous on $[0, T]$.

(ii) $\theta$ is symmetric with respect to $(s, t), \theta(s, s)=0$ for $s \in[0, T]$, and $\lim _{\delta \downarrow 0} \sup \{\theta(t, s):|t-s| \leqq \delta\}=0$.

Note that if $\theta$ is continuous on $[0, T] \times[0, T]$, then $\theta \in \mathscr{C}$ iff $\theta$ is symmetric and $\theta(s, s)=0$ for $s \in[0, T]$. Given a $\theta \in \mathscr{C}$, define $\rho:[0, T] \rightarrow[0, \infty)$ by

$$
\rho(r)=\sup \{\theta(t, s): s, t \in[0, T] \text { and }|t-s| \leqq r\} \text { for } r \in[0, T] .
$$

The function $\rho$ is bounded, nonnegative and nondecreasing on $[0, T]$, and $\lim _{r \downarrow} \rho(r)=0$. Hence we may assume without loss of generality that $\rho$ is right-continuous on $[0, T)$. If $\theta$ is continuous on $[0, T] \times[0, T]$, then $\rho$ is 
upper semicontinuous on $[0, T]$ and right-continuous on $[0, T)$. Let $\rho:[0, T]$ $\rightarrow \boldsymbol{R}$ be any bounded, nonnegative, nondecreasing and right-continuous function with $\lim _{r \downarrow 0} \rho(r)=\rho(0)=0$. Then the function $\theta$ defined by $\theta(t, s)=\rho(|t-s|)$ for $(t, s) \in[0, T] \times[0, T]$ belongs to the class $\mathscr{C}$.

A function $\theta$ defined on all of $[0, T] \times[0, T]$ is said to belong to the class $\mathscr{C}_{B V}$ if $\theta \in \mathscr{C}$ and the following additional condition holds:

(iii) There exists a constant $M \geqq 0$ such that $\sum_{k=1}^{N} \theta\left(t_{k}, t_{k-1}\right) \leqq M$ for all partitions $\Delta=\left\{0=t_{0}<t_{1}<\cdots<t_{N}=T\right\}$ of $[0, T]$.

\section{§ 2. Basic Hypotheses and Main Results}

We begin by making a basic assumption on the Fréchet space $X$. Let $\mathfrak{p}: X \rightarrow[0, \infty]$ be a fixed l.s.c. seminorm on $X$ and let $\left\{X_{\alpha}: \alpha>0\right\}$ and $X_{0}$ be defined by (1.2). In what follows, we suppose that there exists a Banach space $\left(Y,\|\cdot\|_{Y}\right)$ and a family $\left\{P_{\alpha}: \alpha>0\right\}$ of injective linear operators from $X_{0}$ into $Y$. Hence, for each $\alpha>0$, the functional $\|\cdot\|_{\alpha}: X_{0} \rightarrow[0, \infty)$ defined by

$$
\|v\|_{\alpha}=\left\|P_{\alpha} v\right\|_{Y} \quad \text { for } \quad v \in X_{0}
$$

gives a norm on $X_{0}$. We then assume that each of the p-bounded absolutely convex subsets $X_{\alpha}$ is embedded into the Banach space $Y$ through the family $\left\{P_{\alpha}\right\}$ of operators in the following sense:

(F) For each $\alpha>0$, the relative topology (induced by the metric topology of $X$ ) on the closed convex subset $X_{\alpha}$ is equivalent to the metric topology on $X_{\alpha}$ defined by the metric $d_{\alpha}(v, w)=\|v-w\|_{\alpha}, v, w \in X_{\alpha}$.

Under condition $(\mathbf{F})$ we put three conditions on each of the operators $A(t), t \in[0, T]$, namely:

(A.1) The domain $D(A(t))$ and the range $R(A(t))$ are both contained in $X_{0}$ and $D(A(t)) \cap X_{\alpha}$ is dense in $X_{\alpha}$ for $\alpha>0$.

(A.2) There exist constants $\omega \geqq 0$ and $\lambda_{0} \in(0,1 / \omega)$ such that for each $\alpha>0$ the inequality

$$
(1-\omega \lambda)\|v-w\|_{\alpha} \leqq\left\|\left(v-\lambda v^{\prime}\right)-\left(w-\lambda w^{\prime}\right)\right\|_{\alpha}
$$

holds for $v, w \in X_{\alpha} \cap D(A(t)), v^{\prime} \in A(t) v, w^{\prime} \in A(t) w$, and $\lambda \in\left(0, \lambda_{0}\right)$.

(A.3) There exists a constant $\lambda_{0} \in(0,1 / \omega)$ such that the range $R(I-\lambda A(t))$ of the operator $I-\lambda A(t)$ coincides with $X_{0}$ for $\lambda \in\left(0, \lambda_{0}\right)$.

Condition (A.2) states that for each $\alpha>0$ the operator $A(t)-\omega I$ is dissipative on $X_{\alpha}$ with respect to the norm $\|\cdot\|_{\alpha}$; and conditions (A.2) and (A.3) together imply that for $t \in[0, T]$ and $\lambda \in\left(0, \lambda_{0}\right)$ the operator $I-\lambda A(t)$ is injective and the resolvent

$$
J(\lambda ; t)=(I-\lambda A(t))^{-1}
$$


exists as a single-valued operator with domain $X_{0}$ and range contained in $X_{0}$. Hereafter we denote by $A(t ; \lambda)$ the Yosida approximation of $A(t)$,

$$
A(t ; \lambda)=\lambda^{-1}[J(\lambda ; t)-I] .
$$

We then put three conditions on the family $\{A(t)\}$. First, the resolvent $J(\lambda ; t)$ is assumed to be stable with respect to $\mathfrak{p}$ in the following sense:

(S.1) There exist constants, $a, a^{\prime} \geqq 0$ and $\lambda^{*} \in\left(0, \min \left\{\lambda_{0}, 1 / a\right\}\right)$ such that

$$
\mathfrak{p}(J(\lambda ; t) v) \leqq(1-a \lambda)^{-1}\left[\mathfrak{p}(v)+a^{\prime} \lambda\right]
$$

for $\lambda \in\left(0, \lambda^{*}\right), t \in[0, T]$ and $v \in X_{0}$.

Next, we specify the $t$-dependence of $A(t)$. To this end, we divide each of the closed convex sets $X_{\alpha}, \alpha>0$, into closed convex sets by making use of another l.s.c. convex functional, say $\mathfrak{q}_{\alpha}$, satisfying condition (S.2) below, and then impose the uniform $t$-dependence of $A(t)$ on each of the divided closed convex subsets. Recall that $\mathscr{C}$ denotes the class of functions on $[0, T] \times[0, T]$ as introduced in Section 1.

(S.2) For each $\alpha>0$ there exist an l.s.c. convex functional $\mathfrak{q}_{\alpha}: X \rightarrow[0, \infty]$, constants $b_{\alpha}, b_{\alpha}^{\prime} \geqq 0$ and a number $\lambda_{\alpha} \in\left(0, \lambda^{*}\right)$ ( $\lambda^{*}$ being a constant as mentioned in (S.1)) such that $D\left(\mathfrak{q}_{\alpha}\right)$ is dense in $X_{\beta}$ for $\beta \in(0, \alpha]$ and the inequality

$$
\mathfrak{q}_{\alpha}(J(\lambda ; t) v) \leqq \exp \left(b_{\alpha} \lambda\right)\left[\mathfrak{q}_{\alpha}(v)+b_{\alpha}^{\prime} \lambda\right]
$$

holds for $\lambda \in\left(0, \lambda_{\alpha}\right), t \in[0, T]$ and $v \in X_{\alpha} \cap D\left(\mathfrak{q}_{\alpha}\right)$ with $(1-a \lambda)^{-1}\left[\mathfrak{p}(v)+a^{\prime} \lambda\right]<\alpha$.

(S.3) For $\alpha>0$ and $\gamma>0$ there is a function $\theta_{\alpha}^{r}$ in $\mathscr{C}$ such that the inequality

$$
(1-\omega \lambda)\|J(\lambda ; s) v-J(\lambda ; t) v\|_{\alpha} \leqq \lambda \theta_{\alpha}^{r}(s, t)
$$

holds for $s, t \in[0, T], \lambda \in\left(0, \lambda_{\alpha}\right)$ and $v \in X$ satisfying

$$
(1-a \lambda)^{-1}\left[\mathfrak{p}(v)+a^{\prime} \lambda\right]<\alpha \quad \text { and } \exp \left(b_{\alpha} \lambda\right)\left[\mathfrak{q}_{\alpha}(v)+b_{\alpha}^{\prime} \lambda\right]<\gamma .
$$

We have thus intorudced three groups of conditions on both the Fréchet space $X$ and the family of operators $\{A(t)\}$. We then give a few remarks on the conditions and describe how the conditions will be applied to construct an evolution operator on $X_{0}$ which provides the weak solutions as mentioned in the Introduction.

Firstly, conditions (A.2) and (A.3) are modified forms of the typical conditions for the generation of nonlinear semigroups in Banach spaces. See [1]-[3]. With regard to (A.3) it may be more adequate to impose weaker conditions that allow the constants $\omega$ and $\lambda_{0}$ to depend upon $\alpha$. (Hence, for instance, condition (A.3) should be weakened to the condition that for each $\beta>0$ there exist $\alpha \geqq \beta$ and $\lambda_{\alpha}>0$ such that the range of $I-\lambda A(t)_{X_{\alpha}}$ contains $X_{\beta}$ 
for $\lambda \in\left(0, \lambda_{\alpha}\right)$.) In fact, it is possible to treat such general cases by the same method as discussed below. See Section 5. However conditions (A.1), (A.2) and (A.3) are fulfilled by various families of nonlinear operators when appropriate families $\left\{P_{\alpha}\right\}$ of injective operators are chosen (see Section 6 for a typical example); and furthermore conditions of the forms (A.i), $i=1,2,3$, enable us to give a concise exposition of the generation theorem for nonlinear evolution operators under consideration. Hence we employ these conditions here as our basic hypotheses.

Secondly, under conditions (F), (A.1)-(A.3), and (S.1), an approximating discrete scheme for the system $\left\{(D E)_{s}\right\}$ can be constructed in the following way: Let $s \in[0, T), \beta>0$, and let $\alpha>e^{2 a T}\left(\beta+a^{\prime} T\right)$. Then for every $u_{0} \in X_{\beta}$ and every sequence $\left(\Delta_{n}\right)$ of partitions of $[s, T]$ with $\left|\Delta_{n}\right|<\lambda^{*}$ and $\left|\Delta_{n}\right| \rightarrow 0(n \rightarrow \infty)$, one can define a doubly indexed sequence $\left(\left(u_{k}^{n}, v_{k}^{n}\right): 1 \leqq k \leqq N(n), n \geqq 1\right)$ by

$$
u_{k}^{n}=\prod_{p=1}^{k} J\left(h_{p}^{n} ; t_{p}^{n}\right) u_{0} \quad \text { and } \quad v_{k}^{n}=A\left(t_{k}^{n} ; h_{k}^{n}\right) u_{k-1}^{n}
$$

for $1 \leqq k \leqq N(n)$ and $n \geqq 1$, where $\Delta_{n}=\left\{s=t_{0}^{n}<t_{1}^{n}<\cdots<t_{N(n)}^{n}=T\right\}$ and $h_{p}^{n}=t_{p}^{n}-$ $t_{p-1}^{n}$ for $1 \leqq p \leqq N(n)$. For each $n \geqq 1$, the finite sequence $\left(\left(u_{k}^{n}, v_{k}^{n}\right): 1 \leqq k \leqq N(n)\right)$ is well-defined as a sequence in $X_{\alpha} \times X$ by conditions (A.3) and (S.1) and satisfies the difference equation

$((D E))_{n} \quad\left(h_{k}^{n}\right)^{-1}\left[u_{k}^{n}-u_{k-1}^{n}\right]=v_{k}^{n},\left(u_{k}^{n}, v_{k}^{n}\right) \in A\left(t_{k}^{n}\right), 1 \leqq k \leqq N(n) ; u_{0}^{n}=u_{0}$.

The system of difference equations $\left\{((D E))_{n}: n \geqq 1\right\}$ can be regarded as an approximate discrete scheme for $(D E)_{s}$ with $(I C)_{s}$. We now define step functions $u^{n}:[0, T] \rightarrow X, n \geqq 1$, by the equations $u^{n}(s)=u_{0}^{n}$ and

$$
u^{n}(t)=\sum_{k=1}^{N(n)} u_{k}^{n} \chi_{\left(t_{k-1}^{n}, t_{k}^{n}\right]}(t) \text { for } t \in(s, T] .
$$

The sequence $\left(u^{n}\right)$ of step functions may be regarded as a sequence of approximate solutions to $(D E)_{s}$; and if there is an $X$-valued, strongly continuous function $u$ on $[s, T]$ and if the convergence

$$
u(t)=\lim _{n \rightarrow \infty} u^{n}(t)
$$

holds in the space $\mathscr{B}([s, T] ; X)$ of $X$-valued, bounded functions on $[s, T]$, then the limit function $u$ gives a weak solution of $(D E)_{s}$. For, if a sequence $\left(u_{n}\right)$ of piecewise linear functions in the space $\mathscr{C}([s, T] ; X)$ of $X$-valued, continuous functions on $[s, T]$ is defined by the equations $u_{n}(s)=u_{0}$ and

$$
u_{n}(t)=\left(h_{k}^{n}\right)^{-1}\left[\left(t-t_{k-1}^{n}\right) u_{k}^{n}+\left(t_{k}^{n}-t\right) u_{k-1}^{n}\right]
$$

for $t \in\left(t_{k-1}^{n}, t_{k}^{n}\right]$ and $1 \leqq k \leqq N(n)$, and if the convergence (2.5) holds for an $X$ valued function $u$ in $\mathscr{C}([s, T] ; X)$, then $u_{n}$ converges in $\mathscr{C}([s, T] ; X)$ to $u$. 
Notice that the sequence $\left(u_{n}(\cdot)\right)$ satisfies rather strong conditions in the sense the $\left(\mathbf{w}_{1}\right)$ through $\left(\mathbf{w}_{3}\right)$ hold with $C=X_{\alpha}$ and $f_{n} \equiv 0(n \geqq 1)$.

Thus the crucial step for constructing the desired evolution operator $\mathscr{U}$ is the verification of the convergence (2.5). By virtue of condition (F) the convergence problem for the solution $\left(u_{k}^{n}\right)$ of $((D E))_{n}$ is passed on through the operator $P_{\alpha}$ to that for the sequence $\left(P_{\alpha} u_{k}^{n}\right)$ in the Banach space $Y$. We then aim to apply the same type of convergence argument as in [7], Section 3 , to the sequence $\left(P_{\alpha} u_{k}^{n}\right)$ by imposing conditions $(\mathbf{F}),(\mathbf{A . i})$ and (S.i).

Thirdly, condition (S.3) should be understood to be a generalized form of the conditions (C.1) and (C.2) as mentioned in Crandall-Pazy [3]. See also [7], Section 1. Now in view of condition (S.2) we define closed convex subsets $X_{\alpha}^{\gamma}, \alpha>0, \gamma>0$, by

$$
X_{\alpha}^{\gamma} \equiv\left\{v \in X_{\alpha}: \mathfrak{q}_{\alpha}(v) \leqq \gamma\right\}=\left\{v \in X: \mathfrak{p}(v) \leqq \alpha, \mathfrak{q}_{\alpha}(v) \leqq \gamma\right\} .
$$

Let $\beta>0, \delta>0, \alpha>\beta$ and let $v \in X_{\alpha}^{\delta} \cap X_{\beta}$. Then we see from (A.2), (A.3) and (S.1) that the mapping $\lambda \rightarrow A(t ; \lambda) v$ defines an $X$-valued function on some interval $\left(0, \lambda_{\alpha}^{\prime}\right)$ such that the function $\lambda \rightarrow(1-\lambda \omega)\|A(t ; \lambda) v\|_{\alpha}$ is monotone nonincreasing on $\left(0, \lambda_{\alpha}^{\prime}\right)$ (cf. Crandall [1], p. 435). We set

$$
|A(t) v|_{\alpha}=\lim _{\lambda \downarrow 0}\|A(t ; \lambda) v\|_{\alpha} \quad \text { for } \quad v \in X_{\beta} \cap X_{\alpha}^{\delta}
$$

and define

$$
\hat{D}(\beta ; \alpha, \delta)=\left\{v \in X_{\beta} \cap X_{\alpha}^{\delta}:|A(t) v|_{\alpha}<\infty\right\} .
$$

Clearly, $X_{\beta} \cap X_{\alpha}^{\delta} \cap D(A(t)) \subset \hat{D}(\beta ; \alpha, \delta) \subset X_{\beta} \cap X_{\alpha}^{\delta}$. Further, the set $\hat{D}(\beta ; \alpha, \gamma)$ is independent of $t$. In fact, let $s, t \in[0, T]$ and let $v \in X_{\beta} \cap X_{\alpha}^{\delta}$ satisfy $|A(s) v|_{\alpha}<\infty$. Then condition (S.3) implies that $|A(t) v|_{\alpha} \leqq|A(s) v|_{\alpha}+\theta_{\alpha}^{r}(t, s) \leqq|A(s) v|_{\alpha}+\rho_{\alpha}^{r}(T)$, where $\rho_{\alpha}^{r}$ is the function defined through (1.3) for $\theta_{\alpha}^{r}$. This means that $|A(s) v|_{\alpha}$ $<\infty$ iff $|A(t) v|_{\alpha}<\infty$.

We are now in a position to state our main theorem.

TheOREM 1. Under conditions (F), (A.1) and (S.i), $i=1,2,3$, there exists an evolution operator $\mathscr{U}$ on $X_{0}$ with the following properties:

(a) Given an $s \in[0, T), v \in X_{0}$ and a sequence $\left\{\Delta_{n}\right\}$ of pariitions of $[s, T]$ with $\left|\Delta_{n}\right| \rightarrow 0$, we have the convergence

$$
U(t, s) v=\lim \prod_{p=1}^{k}\left[I-\left(t_{p}^{n}-t_{p-1}^{n}\right) A\left(t_{p}^{n}\right)\right]^{-1} v \quad \text { for } \quad t \in[s, T],
$$

where the limit is taken in the metric topology of $X$ as $n \rightarrow \infty$ and $t_{k}^{n} \rightarrow t$.

(b) For $\beta>0, \alpha>e^{2 a T}\left(\beta+a^{\prime} T\right), s \in[0, T)$ and $v \in X_{\beta}$, the $X$-valued function $u(t) \equiv U(t, s) v$ gives a unique weak solution with the initial-value $v$ of $(D E)_{s}$ constrained in $X_{\alpha}$. 
(c) Given $s, t \in[0, T]$ with $s \leqq t$, the operator $U(t, s)$ is continuous in the sense that for $\beta>0$ and $\alpha>e^{2 a T}\left(\beta+\alpha^{\prime} T\right)$, we have

$$
\|U(t, s) v-U(t, s) w\|_{\alpha} \leqq e^{\omega(t-s)}\|v-w\|_{\alpha} \quad \text { for } \quad v, w \in X_{\beta} .
$$

Therefore, for each $\beta>0$, the family of operators $\{U(t, s)\}$ is equicontinuous on $X_{\beta}$ with respect to the metric topology of $X$.

(d) For $v \in X_{0}$ and $s, t \in[0, T]$ with $s \leqq t$,

$$
\mathfrak{p}(U(t, s) v) \leqq e^{a(t-s)}\left[\mathfrak{p}(v)+a^{\prime}(t-s)\right]
$$

(e) Let $\beta, \delta>0, \alpha>\exp (2 a T)\left(\beta+a^{\prime} T\right)$, and let $\gamma>\exp \left(b_{\alpha} T\right)\left(\delta+b_{\alpha}^{\prime} T\right)$. Then the inequality

$$
\mathfrak{q}_{\alpha}(U(t, s) v) \leqq \exp \left[b_{\alpha}(t-s)\right]\left[\mathfrak{q}_{\alpha}(v)+b_{\alpha}^{\prime}(t-s)\right] \leqq \gamma
$$

holds for $0 \leqq s \leqq t \leqq T$ and $v \in X_{\beta} \cap X_{\alpha}^{\delta}$.

(f) Let $\beta>0, \delta>0, \alpha>\exp (2 a T)\left(\beta+a^{\prime} T\right)$, and let $\gamma \geqq \exp \left(b_{\alpha} T\right)\left(\alpha+b_{\alpha}^{\prime} T\right)$. Then $\alpha>\beta$ and $\hat{D}\left(\beta^{\prime} ; \alpha, \delta^{\prime}\right)$ is dense in $X_{\beta} \cap X_{\alpha}^{\delta}$ for $\delta^{\prime}>\delta$ and $\alpha>\beta^{\prime}>\beta$, where $\hat{D}\left(\beta^{\prime} ; \alpha, \delta^{\prime}\right)$ is the set defined by $(2.8)$ with $\beta$ and $\delta$ replaced respectively by $\beta^{\prime}$ and $\delta^{\prime}$. If $v \in \hat{D}(\beta ; \alpha, \delta), \theta_{\alpha}^{\gamma}$ is the function specified for the $\alpha$ and $\gamma$ by (S.3), and if $\theta_{\alpha}^{r} \in \mathscr{C}_{\boldsymbol{B V}}$, then the weak solution $u(t) \equiv U(t, s) v$ with initial-value $v$ of $(D E)_{s}$ is Lipschitz continuous over $[s, T]$ relative to the norm $\|\cdot\|_{\alpha}$.

\section{$\S 3 . \quad$ Basic Estimates}

In this section we prepare some basic estimates which will be used in the proof of Theorem 1. First conditions (A.2), (S.1) and (S.3) together yield

$$
(1-\omega \lambda)\|J(\lambda ; s) v-J(\lambda ; t) w\|_{\alpha} \leqq\|v-w\|_{\alpha}+\lambda \theta_{\alpha}^{\gamma}(s, t)
$$

for $s, t \in[0, T], \lambda \in\left(0, \lambda_{\alpha}\right)$ and $v, w$ in $X_{\alpha}^{r}$ satisfying (2.3). In view of this we obtain the following lemma.

Lemma 3.1. Let $\alpha>0, \gamma>0$ and $\lambda, \mu \in\left(0, \lambda_{\alpha}\right)$. Let $s, t \in[0, T]$. Then for $u$, $v$ satisfying (2.3) one has the estimate

$$
\begin{aligned}
& (\lambda+\mu-\lambda \mu \omega)\|J(\lambda ; s) u-J(\mu ; t) v\|_{\alpha} \\
& \quad \leqq \lambda\|J(\lambda ; s) u-v\|_{\alpha}+\mu\|J(\mu ; t) v-u\|_{\alpha}+3 \lambda \mu \exp [\omega(\lambda+\mu)] \theta_{\alpha}^{r}(t, s) .
\end{aligned}
$$

Proof. We may assume without loss of generality that $\mu \leqq \lambda$. Since $A(t)-\omega I$ is dissipative on $X_{\alpha}$ with respect to the norm $\|\cdot\|_{\alpha}$, the inequality

$$
\begin{aligned}
& (\lambda+\mu-\lambda \mu \omega)\|J(\lambda ; s) u-J(\mu ; s) v\|_{\alpha} \\
& \quad \leqq \lambda\|J(\lambda ; s) u-v\|_{\alpha}+\mu\|J(\mu ; t) v-u\|_{\alpha}+(1-\omega \mu)^{-1} \mu^{2} \theta_{\alpha}^{r}(t, s)
\end{aligned}
$$


is obtained. In fact, $0 \leqq \lambda \mu\left(-w+z, x^{*}\right)+\lambda \mu \omega\|J(\lambda ; s) u-J(\mu ; s) v\|_{\alpha}^{2}$ for $w \epsilon$ $A(s) J(\lambda ; s) u, z \in A(s) J(\mu ; s) v$, and some $x^{*} \in F_{\alpha}(J(\lambda ; s) u-J(\mu ; s) v)$ by (A.2), where $F_{\alpha}$ denotes the duality mapping of the normed space $\left(X_{0},\|\cdot\|_{\alpha}\right)$ and $\left(z, x^{*}\right)$ denotes the value of the functional $x^{*}$ at the point $z$. Hence we have

$$
\begin{aligned}
(\lambda+\mu) & \|J(\lambda ; s) u-J(\mu ; s) v\|_{\alpha}^{2} \leqq \lambda\left(J(\lambda ; s) u-J(\mu ; s) v+\mu z, x^{*}\right) \\
& +\mu\left(J(\lambda ; s) u-J(\mu ; s) v-\lambda w, x^{*}\right)+\lambda \mu \omega\|J(\lambda ; s) u-J(\mu ; s) v\|_{\alpha}^{2} \\
\leqq & {\left[\lambda\|J(\lambda ; s) u-v\|_{\alpha}+\mu\|J(\lambda ; s) v-u\|_{\alpha}+\lambda \mu \omega\|J(\lambda ; s) u-J(\mu ; s) v\|_{\alpha}\right] } \\
& \times\|J(\lambda ; s) u-J(\mu ; s) v\|_{\alpha} .
\end{aligned}
$$

Combining this with condition (S.3), we obtain the above inequality. On the other hand, (3.1) yields $\|J(\lambda ; s) u-J(\mu ; t) v\|_{\alpha} \leqq\|J(\lambda ; s) u-J(\mu ; s) v\|_{\alpha}+(1-\omega \mu)^{-1}$ $\times \mu \theta_{\alpha}^{r}(t, s)$. So, noting that $(1-\omega \mu)^{-1}\left[\mu^{2}+\mu(\lambda+\mu-\lambda \mu \omega)\right] \leqq 3 \lambda \mu \exp (2 \omega \mu)$, we obtain the desired estimate.

Next, we need the following lemma.

Lemma 3.2. Let $r \in[0, T], \beta>0, \alpha>\beta$, and let $\nu(\alpha, \beta)=\min \left\{\lambda^{*},(\alpha-\beta) /\right.$ $\left.\left(a \alpha+a^{\prime}\right)\right\}$. Let $\nu \in(0, \nu(\alpha, \beta)), z \in X_{\beta}$, and $z_{\nu}=J(\nu ; r) z$. Then $\left\|z_{\nu}-z\right\|_{\alpha} \rightarrow 0$ as $\nu \rightarrow 0$.

Proof. First assume that $z \in X_{\beta} \cap D(A(r))$. Then $z_{\nu} \in X_{\alpha} \cap D(A(r))$ by (S.1); hence $(1-\omega \nu)\left\|z_{\nu}-z\right\|_{\alpha} \leqq \nu \cdot \inf \left\{\|w\|_{\alpha}: w \in A(r) z\right\}$ for $\nu \in(0, \nu(\alpha, \beta))$ by condition (A.2). Next, assume that $z \in X_{\beta}$. Then it follows from (A.1) that there exists a sequence $\left(z_{n}\right)$ in $X_{\beta} \cap D(A(r))$ such that $z_{n} \rightarrow z$ in $X_{\beta}$ with respect to the relative topology on $X_{\beta}$ induced by the metric topology of $X$. Hence $z_{n} \rightarrow z$ in the sense of the relative topology on $X_{\alpha}$, so that $\left\|z_{n}-z\right\|_{\alpha} \rightarrow 0$ as $n \rightarrow \infty$ by condition $(\mathbf{F})$. Since $J(\nu ; r) z$ as well as $J(\nu ; r) z_{n}$ belong to $X_{\alpha}$ for $n \geqq 1$, condition (A.2) implies that $\left\|\boldsymbol{z}_{\nu}-\boldsymbol{z}\right\|_{\alpha} \leqq\left\|J(\nu ; r) z-J(\nu ; r) z_{n}\right\|_{\alpha}+\left\|J(\nu ; r) z_{n}-\bar{z}_{n}\right\|_{\alpha}$ $+\left\|z_{n}-z\right\|_{\alpha} \leqq(1-\omega \nu)^{-1} 2\left\|z_{n}-z\right\|_{\alpha}+\left\|J(\nu ; r) z_{n}-z_{n}\right\|_{\alpha}$ for $n \geqq 1$ and $\nu \in(0, \nu(\alpha, \beta))$. From this and the above-mentioned we infer that $\left\|z_{\nu}-z\right\|_{\alpha} \rightarrow 0$ as $\nu \downarrow 0$. q.e.d.

In order to obtain the proof of Theorem 1, it is crucial to establish Assertion (a). To that end, let $s \in[0, T), \beta>0, \alpha>e^{2 a T}\left(\beta+a^{\prime} T\right), \delta>0$, and let $\gamma>\exp \left(b_{\alpha} T\right)\left(\delta+b_{\alpha}^{\prime} T\right)$, where $a, a^{\prime}, b_{\alpha}, b_{\alpha}^{\prime}$ are the constants provided by (S.1) and (S.2). Let $u_{0} \in X_{\beta}^{\delta}$ and choose a sequence of partitions of [s,T], $\Delta_{n}=\left\{s=t_{0}^{n}<t_{1}^{n}<\cdots<t_{N(n)}^{n}=T\right\}, n \geqq 1$, so that $\left|\Delta_{n}\right|<\lambda_{\alpha} / 2$ and $\left|\Delta_{n}\right| \rightarrow 0$ (notice that $\left.\left|\Delta_{n}\right|<\min \left\{1 / 2 a, \lambda_{0}, \lambda_{\alpha}, T\right\}\right)$. Then for each $n \geqq 1$ one can define a sequence $\left(\left(u_{k}^{n}, v_{k}^{n}\right): 1 \leqq k \leqq N(n)\right)$ by

$$
u_{0}^{n}=u_{0}, u_{k}^{n}=\prod_{p=1}^{k} J\left(h_{p}^{n} ; t_{p}^{n}\right) u_{0} \quad \text { and } \quad v_{k}^{n}=\left(h_{k}^{n}\right)^{-1}\left[u_{k}^{n}-u_{k-1}^{n}\right]
$$

where $h_{p}^{n}=t_{p}^{n}-t_{p-1}^{n}, 1 \leqq p \leqq N(n)$. Since 


$$
\begin{aligned}
& \mathfrak{p}\left(u_{k}^{n}\right) \leqq \prod_{p=1}^{k}\left(1-a h_{p}^{n}\right)^{-1}\left[\mathfrak{p}\left(u_{0}\right)+\left(t_{k}^{n}-s\right) a^{\prime}\right] \leqq \alpha, \\
& \mathfrak{q}_{\alpha}\left(u_{k}^{n}\right) \leqq \exp \left[b_{\alpha}\left(t_{k}^{n}-s\right)\right]\left[\mathfrak{q}_{\alpha}\left(u_{0}\right)+b_{\alpha}^{\prime}\left(t_{k}^{n}-s\right)\right] \leqq \gamma,
\end{aligned}
$$

by (S.1) and (S.2), it follows that the sequence $\left(u_{k}^{n}\right)$ lies in $X_{\alpha}^{\gamma}$. We then establish an important estimate for $\left(u_{k}^{n}\right)$ by applying Lemma 3.1.

Let $\alpha, \beta, \delta$ and $\gamma$ be as specified above. Let $s \in[0, T), \hat{s} \in[0, T)$ and let $\left\{\Delta_{n}\right\},\left\{\hat{\Delta}_{m}\right\}$ be, respectively, sequences of partitions of the intervals $[s, T]$ and $[\hat{s}, T]$ such that $\left|\Delta_{n}\right|,\left|\hat{\Delta}_{m}\right|<\lambda_{\alpha} / 2,\left|\Delta_{n}\right| \rightarrow 0$ and $\left|\hat{\Delta}_{m}\right| \rightarrow 0$. We write $\Delta_{n}=\left\{s=t_{0}^{n}<t_{1}^{n}\right.$ $\left.<\cdots<t_{N(n)}^{n}=T\right\}, \hat{\Delta}_{m}=\left\{\hat{s}=\hat{t}_{0}^{m}<\hat{t}_{1}^{m}<\cdots<\hat{t}_{\hat{N}(m)}^{m}=T\right\}, h_{p}^{n}=t_{p}^{n}-t_{p-1}^{n}$ for $1 \leqq p \leqq N(n)$, and $\hat{h}_{q}^{m}=\hat{t}_{q}^{m}-\hat{t}_{q-1}^{m}$ for $1 \leqq q \leqq \hat{N}(m)$ and $m, n \geqq 1$. Now let $u_{0}, \hat{u}_{0} \in X_{\beta} \cap \bar{X}_{\alpha}^{o}$ and then define

$$
\begin{aligned}
& u_{0}^{n}=u_{0}, \quad \hat{u}_{0}^{m}=\hat{u}_{0}, \\
& u_{k}^{n}=\prod_{p=1}^{k} J\left(h_{p}^{n} ; t_{p}^{n}\right) u_{0} \quad \text { and } \quad \hat{u}_{j}^{m}=\prod_{q=1}^{j} J\left(\hat{h}_{q}^{m}: \hat{t}_{q}^{m}\right) \hat{u}_{0}
\end{aligned}
$$

for $1 \leqq k \leqq N(n)$ and $1 \leqq j \leqq \hat{N}(m)$. We are going to estimate the difference between $u_{k}^{n}$ and $\hat{u}_{j}^{m}$ under the conditions imposed in Section 2.

Let $m, n$ be fixed and put

$$
\begin{aligned}
& \alpha_{k, j}=\frac{\hat{h}_{j}^{m}}{h_{k}^{n}+\hat{h}_{j}^{m}}, \quad \beta_{k, j}=\frac{h_{k}^{n}}{h_{k}^{n}+\hat{h}_{j}^{m}}, \quad \gamma_{k, j}=\frac{h_{k}^{n} \hat{h}_{j}^{m}}{h_{k}^{n}+\hat{h}_{j}^{m}}, \\
& a_{k, j}=\left\|u_{k}^{n}-\hat{u}_{j}^{m}\right\|_{\alpha} \quad \text { and } \quad d_{k, j}=3 \exp \left(\omega \lambda_{\alpha}\right) \theta_{\alpha}^{r}\left(t_{k}^{n}, \hat{t}_{j}^{m}\right)
\end{aligned}
$$

for $0 \leqq k \leqq N(n)$ and $0 \leqq j \leqq \hat{N}(m)$, where $\theta_{\alpha}^{r}$ is the function stated in (3.1). Since $u_{k}^{n}$ and $\hat{u}_{j}^{m}$ are all contained in $X_{\alpha}^{r}$, Lemma 3.1 (with $u=u_{k-1}^{n}, v=\hat{u}_{j-1}^{m}, \lambda=h_{k}^{n}$, $\mu=\hat{h}_{j}^{m}, s=t_{k}^{n}$ and $t=\hat{t}_{j}^{m}$ ) yields the inequality

$$
a_{k, j} \leqq \alpha_{k, j} a_{k-1, j}+\beta_{k, j} a_{k, j-1}+\gamma_{k, j}\left(a_{k, j} \omega+d_{k, j}\right) .
$$

Next, take $r \in[0, T], z \in X_{\beta} \cap X_{\alpha}^{\gamma}, \nu \in\left(0, \min \left\{\lambda_{\alpha} / 2, T\right\}\right)$, and put $z_{\nu}=J(\nu ; r) z$ and $w_{\nu}=A(r ; \nu) z$ (hence $\left(z_{\nu}, w_{\nu}\right) \in A(r)$ and $\left.z_{\nu} \in X_{\alpha}^{r}\right)$. Then the application of Lemma 3.1 (with $u=u_{k-1}^{n}, v=z, \lambda=h_{k}^{n}, \mu=\nu, s=t_{k}^{n}$ and $t=r$ ) yields

$$
\left(1-\omega h_{k}^{n}\right)\left\|u_{k}^{n}-z_{\nu}\right\|_{\alpha} \leqq\left\|u_{k-1}^{n}-z_{\nu}\right\|_{\alpha}+h_{k}^{n}\left[\left\|w_{\nu}\right\|_{\alpha}+3 \exp \left(\omega \lambda_{\alpha}\right) \rho_{\alpha}^{\gamma}(T)\right]
$$

for $1 \leqq k \leqq N(n)$, where $\rho_{\alpha}^{r}$ is defined for $\theta_{\alpha}^{r}$ by (1.3). (Here we have used the relations $z_{\nu}-z=\nu w_{\nu}$ and $\left.\left\|u_{k}^{n}-z\right\|_{\alpha} \leqq\left\|u_{k}^{n}-z_{\nu}\right\|_{\alpha}+\nu\left\|w_{\nu}\right\|_{\alpha}\right)$. Also, the same estimates as above are obtained for $\left\{\hat{u}_{j}^{m}\right\}$ and $\left\{\hat{h}_{j}^{m}\right\}$. From this we obtain

$$
\begin{gathered}
\prod_{p=1}^{k}\left(1-\omega h_{p}^{n}\right)\left\|u_{k}^{n}-z_{\nu}\right\|_{\alpha} \leqq\left\|u_{0}-z_{\nu}\right\|_{\alpha}+\left(t_{k}^{n}-s\right)\left[\left\|w_{\nu}\right\|_{\alpha}+3 \exp \left(\omega \lambda_{\alpha}\right) \rho_{\alpha}^{r}(T)\right], \\
\prod_{q=1}^{j}\left(1-\omega \hat{h}_{q}^{m}\right)\left\|\hat{u}_{j}^{m}-z_{\nu}\right\|_{\alpha} \leqq\left\|\hat{u}_{0}-z_{\nu}\right\|_{\alpha}+\left(\hat{t}_{j}^{m}-\hat{s}\right)\left[\left\|w_{\nu}\right\|_{\alpha}+3 \exp \left(\omega \lambda_{\alpha}\right) \rho_{\alpha}^{r}(T)\right]
\end{gathered}
$$


for $1 \leqq k \leqq N(n)$ and $1 \leqq j \leqq \hat{N}(m)$.

Given a pair $j, k$ with $1 \leqq j \leqq \hat{N}(m)$ and $1 \leqq k \leqq N(n)$ and $\eta \in R$, we define

$$
c_{k, j}(\eta)=\left[\left(t_{k}^{n}-\hat{t}_{j}^{m}-\eta\right)^{2}+\left|\Delta_{n}\right|\left(t_{k}^{n}-s\right)+\left|\hat{\Delta}_{m}\right|\left(\hat{t}_{j}^{m}-\hat{s}\right)\right]^{1 / 2} .
$$

Then we see in a way similar to Kobayashi [8], p. 649, that the inequality

$$
\alpha_{k, j} c_{k-1, j}(\eta)+\beta_{k, j} c_{k, j-1}(\eta) \leqq c_{k, j}(\eta)
$$

holds for $1 \leqq k \leqq N(n)$ and $1 \leqq j \leqq \hat{N}(m)$. Moreover, recalling that $\rho_{\alpha}^{\gamma}$ is monotone non-decreasing over $[0, T]$, we infer that if $0<\kappa<\delta \leqq T$ and $r^{\prime} \in[0, \delta-\kappa)$ then the function $\rho_{\alpha}^{\gamma}$ satisfies the inequality

$$
\rho_{\alpha}^{\gamma}(r) \leqq \kappa^{-1} \rho_{\alpha}^{\gamma}(T)\left|r-r^{\prime}\right|+\rho_{\alpha}^{\gamma}(\delta) \quad \text { for } \quad r \in[0, T] .
$$

Using the estimates (3.5) through (3.8), we obtain the following lemma.

Lemma 3.3. Let $0 \leqq|\eta|<\delta \leqq T, 0<\kappa<\delta-|\eta|$, and let $m, n$ be such that $\left|\Delta_{n}\right|$, $\left|\hat{\Delta}_{m}\right|<\min \left\{\delta-|\eta|-\kappa, \lambda_{\alpha} / 2\right\}$. Then, given $r \in[0, T]$ and $\nu \in\left(0, \lambda_{\alpha} / 2\right)$, one has

$$
\begin{aligned}
& \prod_{p=1}^{k}\left(1-h_{p}^{n} \omega\right) \prod_{q=1}^{j}\left(1-\hat{h}_{q}^{m} \omega\right) a_{k, j} \\
& \leq\left\|u_{0}-z_{\nu}\right\|_{\alpha}+\left\|z_{\nu}-\hat{u}_{0}\right\|_{\alpha}+c_{k, j}(s-\hat{s})\left[\left\|w_{\nu}\right\|_{\alpha}+3 \exp \left(\omega \lambda_{\alpha}\right) \rho_{\alpha}^{r}(T)\right] \\
& \quad+3 \exp \left(\omega \lambda_{\alpha}\right)\left(\hat{t}_{j}^{m}-\hat{s}\right)\left[\kappa^{-1} \rho_{\alpha}^{\gamma}(T) c_{k, j}(\eta)+\rho_{\alpha}^{r}(\delta)\right]
\end{aligned}
$$

for $0 \leqq k \leqq N(n)$ and $0 \leqq j \leqq \hat{N}(m)$, where $\prod_{p=1}^{0}\left(1-\omega h_{p}^{n}\right)=\prod_{q=1}^{0}\left(1-\omega \hat{h}_{q}^{m}\right)=1$.

Proof. First (3.6) asserts that the estimate (3.9) holds for the pairs $(k, j)$ with $0 \leqq k \leqq N(n), 0 \leqq j \leqq \hat{N}(m)$ and $k j=0$. Next, let $1 \leqq k \leqq N(n), 1 \leqq j \leqq \hat{N}(m)$, and suppose that (3.9) holds for the pairs $(k-1, j)$ and $(k, j-1)$. We will show that (3.9) holds for the pair $(k, j)$. Once this is done, then it is concluded by induction that (3.9) holds for all pairs $(k, j)$ with $0 \leqq k \leqq N(n)$ and $0 \leqq j \leqq \hat{N}(m)$. Using (3.5), one has

$$
\begin{aligned}
& \omega_{k, j}\left(1-\gamma_{k, j} \omega\right) a_{k, j} \leqq\left(1-\hat{h}_{k}^{n}\right) \alpha_{k, j} \omega_{k-1, j} a_{k-1, j} \\
& \quad+\left(1-\hat{h}_{j}^{m} \omega\right) \beta_{k, j-1} \omega_{k, j-1} a_{k, j-1}+\omega_{k, j} \gamma_{k, j} d_{k, j}
\end{aligned}
$$

where $\omega_{k, j}=\prod_{p=1}^{k}\left(1-h_{p}^{n} \omega\right) \prod_{q=1}^{j}\left(1-\hat{h}_{q}^{m} \omega\right)$. Since $1-\gamma_{k, j} \omega \geqq \max \left\{1-h_{k}^{n} \omega, 1-\right.$ $\left.\hat{h}_{j}^{m} \omega, \omega_{k, j}\right\}$, the above estimate yields

$$
\omega_{k, j} a_{k, j} \leqq \alpha_{k, j} \omega_{k-1, j} a_{k-1, j}+\beta_{k, j} \omega_{k, j-1} a_{k, j-1}+\gamma_{k, j} d_{k, j} .
$$

Hence, by the induction hypothesis, $\omega_{k, j} a_{k, j}$ is bounded by

$$
\begin{aligned}
& \left\|u_{0}-\boldsymbol{z}_{\nu}\right\|_{\alpha}+\left\|\boldsymbol{z}_{\nu}-\hat{u}_{0}\right\|_{\alpha}+\left[\alpha_{k, j} c_{k-1, j}+\beta_{k, j} c_{k, j-1}\right]\left(\left\|w_{\nu}\right\|_{\alpha}+3 \exp \left(\omega \lambda_{\alpha}\right) \rho_{\alpha}^{\gamma}(T)\right) \\
& \quad+3 \exp \left(\omega \lambda_{\alpha}\right)\left(\hat{t}_{j}^{m}-\hat{s}\right) \beta_{k, j}\left[\kappa^{-1} \rho_{\alpha}^{\gamma}(T) \tilde{c}_{k-1, j}+\rho_{\alpha}^{\gamma}(\delta)\right] \\
& \quad+3 \exp \left(\omega \lambda_{\alpha}\right)\left(t_{j-1}^{m}-s\right) \beta_{k, j}\left[\kappa^{-1} \rho_{\alpha}^{\gamma}(T) \tilde{c}_{k, j-1}+\rho_{\alpha}^{\gamma}(\delta)\right]+\gamma_{k, j} d_{k, j},
\end{aligned}
$$


where $c_{k, j} \equiv c_{k, j}(s-\hat{s})$ and $\tilde{c}_{k, j} \equiv c_{k, j}(\eta)$. Therefore, the proof is completed if the sum of the above-mentioned terms does not exceed the right side of (3.9). First, applying (3.7) with $\eta=s-\hat{s}$ to the third term, we infer that the third term is not greater than $c_{k, j}(s-\hat{s})\left[\left\|w_{\nu}\right\|_{\alpha}+3 \exp \left(\omega \lambda_{\alpha}\right) \rho_{\alpha}^{r}(T)\right]$. Next, set $r=\left|t_{k}^{n}-\hat{t}_{j}^{m}\right|$ and $r^{\prime}=\left|\eta-\hat{h}_{j}^{m}\right|$ in (3.8). Then

$$
\begin{aligned}
\gamma_{k, j} d_{k, j} & \leqq 3 \exp \left(\omega \lambda_{\alpha}\right) \beta_{k, j} \hat{h}_{j}^{m} \rho_{\alpha}^{\gamma}\left(\left|t_{k}^{n}-\hat{t}_{j}^{m}\right|\right) \\
& \leqq 3 \exp \left(\omega \lambda_{\alpha}\right) \beta_{k, j} \hat{h}_{j}^{m}\left[\kappa^{-1} \rho_{\alpha}^{\gamma}(T) c_{k, j-1}(\eta)+\rho_{\alpha}^{\gamma}(\delta)\right],
\end{aligned}
$$

since $r^{\prime} \leqq|\eta|+\left|\hat{\Delta}_{m}\right|<\delta-\kappa$ and $\left|r-r^{\prime}\right| \leqq\left|t_{k}^{n}-\hat{t}_{j-1}^{m}-\eta\right| \leqq c_{k, j-1}(\eta)$. Hence the sum of the fourth through sixth terms does not exceed the value

$$
3 \exp \left(\omega \lambda_{\alpha}\right)\left(\hat{t}_{j}^{m}-\hat{s}\right)\left[\kappa^{-1} \rho_{\alpha}^{r}(T)\left(\alpha_{k, j} \tilde{c}_{k-1, j}+\beta_{k, j} \tilde{c}_{k, j-1}\right)+\rho_{\alpha}^{r}(\delta)\right],
$$

which is not greater than $3 \exp \left(\omega \lambda_{\alpha}\right)\left(\hat{t}_{j}^{m}-\hat{s}\right)\left[\kappa^{-1} \rho_{\alpha}^{r}(T) c_{k, j}(\eta)+\rho_{\alpha}^{r}(\delta)\right]$ by (3.7). Thus we conclude that (3.9) holds for the pair $(k, j)$.

q.e.d.

\section{$\S 4$. Construction of Evolution Operators.}

In this section we discuss the generation of an evolution operator associated with the system of evolution equations $\left\{(D E)_{s}: s \in[0, T)\right\}$, and we give the proof of Theorem 1 by dividing it into six steps.

Step 1. Let $s \in[0, T), \beta>0, \alpha>e^{2 a T}\left(\beta+a^{\prime} T\right), \delta>0$, and let $\gamma>\exp \left(b_{\alpha} T\right)$. $\cdot\left(\delta+b_{\alpha}^{\prime} T\right)$. Let $u_{0} \in X_{\beta} \cap X_{\alpha}^{\delta}$. In this step we show that there is a function $w(\cdot):[s, T] \rightarrow Y$ such that $w(s)=P_{\alpha} u_{0}$ and $\left\|P_{\alpha} u_{k}^{n}-w(t)\right\|_{Y} \rightarrow 0$ an $n \rightarrow \infty$ and $t_{k}^{n} \rightarrow t$ in $[s, T]$. Let $s=\hat{s}, \eta=0$ and $z=u_{0}=\hat{u}_{0}$ in (3.9) and suppose that $\left(\Delta_{m}\right)$ is the same sequence of partitions as $\left(\Delta_{n}\right)$. Since

$$
\exp \left[-\omega\left(t_{k}^{n}-s\right) /\left(1-\omega\left|\Delta_{n}\right|\right)\right] \exp \left[-\omega\left(t_{j}^{m}-s\right) /\left(1-\omega\left|\Delta_{m}\right|\right)\right] \leqq \omega_{k, j}
$$

for $0 \leqq j \leqq N(m)$ and $0 \leqq k \leqq N(n),(3.9)$ is written as

$$
\begin{aligned}
& \exp \left[-\omega\left(t_{k}^{n}-s\right) /\left(1-\omega\left|\Delta_{n}\right|\right)\right] \exp \left[-\omega\left(t_{j}^{m}-s\right) /\left(1-\omega\left|\Delta_{m}\right|\right)\right]\left\|u^{n}\left(t_{k}^{n}\right)-u^{m}\left(t_{j}^{m}\right)\right\|_{\alpha} \\
& \leqq \\
& \quad+3 u_{0}^{n}-z_{\nu}\left\|_{\alpha}+\right\| u_{0}^{m}-z_{\nu} \|_{\alpha}+c_{k, j}(0)\left[\left\|w_{\nu}\right\|_{\alpha}+3 \exp \left(\omega \lambda_{\alpha}\right) \rho_{\alpha}^{r}(T)\right] \\
& \quad+3 \exp \left(\omega \lambda_{\alpha}\right)\left(t_{j}^{m}-s\right)\left[\kappa^{-1} \rho_{\alpha}^{r}(T) c_{k, j}(0)+\rho_{\alpha}^{r}(\delta)\right],
\end{aligned}
$$

where

$$
\begin{array}{ll}
0<\kappa<\delta \leqq T, & \max \left\{\left|\Delta_{m}\right|,\left|\Delta_{n}\right|\right\}<\min \left\{\delta-\kappa, \lambda_{\alpha} / 2\right\}, \\
\boldsymbol{z}_{\nu}=J(\nu ; r) u_{0}, & 0<\nu<\min \left\{\lambda_{\alpha} / 2, T\right\},
\end{array}
$$

and $u^{n}$ denotes the simple function $\sum_{h=1}^{N(n)} \boldsymbol{u}_{i}^{n} \chi_{\left(t_{k-1,}^{n} t_{k}^{n]}\right.}$ as mentioned before (2.5).

Now let $t \in[s, T]$. Then there exist $j_{m}$ and $k_{n}$ such that $t \in\left(t_{j_{m}-1}^{m} t_{j \S}\right] \cap$ $\left(t_{k_{n}-1}^{n}, t_{k_{n}}^{n}\right]$. Since $\left|t_{k_{n}}^{n}-t_{j_{m}}^{m}\right| \leqq\left|\Delta_{n}\right|+\left|\Delta_{m}\right|$, (4.1) yields 


$$
\begin{aligned}
\exp [- & \left.\omega\left(t-s+\left|\Delta_{n}\right|\right) /\left(1-\omega\left|\Delta_{n}\right|\right)\right] \exp \left[-\omega\left(t-s+\left|\Delta_{m}\right|\right) /\left(1-\omega\left|\Delta_{m}\right|\right)\right]\left\|u^{n}(t)-u^{m}(t)\right\|_{\alpha} \\
\leqq & \left\|u_{0}-\boldsymbol{z}_{\nu}\right\|_{\alpha}+\left\|u_{0}-\boldsymbol{z}_{\nu}\right\|_{\alpha}+3 \exp \left(\omega \lambda_{\alpha}\right)(T-s) \rho_{\alpha}^{\gamma}(\delta) \\
& +\left[3 \exp \left(\omega \lambda_{\alpha}\right) \kappa^{-1} \rho_{\alpha}^{\gamma}(T)(T-s)+\left\|w_{\nu}\right\|_{\alpha}+3 \exp \left(\omega \lambda_{\alpha}\right) \rho_{\alpha}^{\gamma}(T)\right] \\
& \times\left[\left(T-s+\lambda_{\alpha}\right)\left(\left|\Delta_{n}\right|+\left|\Delta_{m}\right|\right)\right]^{1 / 2}
\end{aligned}
$$

for $m$ and $n$ sufficiently large.

Since $\rho_{\alpha}^{\gamma}(\delta) \downarrow 0$ as $\delta \downarrow 0$ and $\left\|\boldsymbol{z}_{\nu}-u_{0}\right\|_{\alpha} \rightarrow 0$ as $\nu \rightarrow 0$ by Lemma 3.2, we infer (by taking $\nu$ and $\delta$ sufficiently small and then letting $m, n \rightarrow \infty$ ) that the limit

$$
w(t)=Y-\lim _{n \rightarrow \infty} P_{\alpha} u^{n}(t)
$$

exists for $t \in[s, T]$ and the convergence is uniform on $[s, T]$ with respect to $t$. Moreover, it is seen from (3.9) that the limit function $w(\cdot)$ does not depend upon the choice of sequences $\left(\Delta_{n}\right)$ of partitions of $[0, T]$.

Step 2. Let $w(\cdot)$ be the limit function obtained by (4.2). Since $u^{n}(t) \in X_{\alpha}$ for $t \in[s, T]$ and $n \geqq 1$ by (3.3), it follows from condition $(\mathbf{F})$ that $w(t) \in P_{\alpha}\left(X_{\alpha}\right)$ for $t \in[s, T]$. In view of this we put

$$
u(t)=P_{\alpha}^{-1} w(t) \quad \text { for } \quad t \in[s, T] .
$$

In this step we demonstrate that the $X$-valued function $u(\cdot)$ is strongly continuous over $[s, T]$. Let $\hat{s}=s,\left(\hat{\Delta}_{m}\right)=\left(\Delta_{n}\right)$, and let $z=\hat{u}_{0}=u_{0}$ in (3.9). Take $t$, $t^{\prime} \in[s, T]$ and $\kappa>0$ satisfying $\eta=t^{\prime}-t,|\eta|<T-s$ and $\kappa+\max \left\{\left|\Delta_{m}\right|,\left|\Delta_{n}\right|\right\}<T-s$ $-|\eta|$, and put $\delta=|\eta|+\kappa+\max \left\{\left|\Delta_{m}\right|,\left|\Delta_{n}\right|\right\}+1 / n$. Apparently, $\eta, \kappa$ and $\delta$ satisfy the conditions imposed in Lemma 3.3. Hence, letting $m, n \rightarrow \infty, t_{j}^{m} \rightarrow t, t_{k}^{n} \rightarrow t^{\prime}$ in (3.9) and then $\kappa \downarrow 0$ in the resultant inequality, we infer with the aid of the convergence (4.2) that

$$
\begin{aligned}
\exp [ & \left.-\omega\left(t+t^{\prime}\right)\right]\left\|w(t)-w\left(t^{\prime}\right)\right\|_{Y} \\
\leqq & 2\left\|u_{0}-z_{\nu}\right\|_{\alpha}+\left|t-t^{\prime}\right|\left(\left\|w_{\nu}\right\|_{\alpha}+3 \exp \left(\omega \lambda_{\alpha}\right) \rho_{\alpha}^{r}(T)\right) \\
& +3 \exp \left(\omega \lambda_{\alpha}\right) t \rho_{\alpha}^{r}\left(\left|t-t^{\prime}\right|+0\right) .
\end{aligned}
$$

Since $\rho_{\alpha}^{r}(r) \downarrow 0$ as $r \downarrow 0$ and $\left\|z_{\nu}-u_{0}\right\|_{\alpha} \rightarrow 0$ as $\nu \downarrow 0$ by Lemma 3.2, (4.4) implies that $w(\cdot)$ is uniformly continuous on $[s, T]$ in the sense of the norm of $Y$. But this means that $u(\cdot):[s, T] \rightarrow X$ is continuous with respect to the norm $\|\cdot\|_{\alpha}$. Moreover, (3.3) and (4.2) together show that for each $t \in[s, T]$ the sequence $\left(u^{n}(t)\right)$ is Cauchy in $X_{\alpha}$ with respect to the norm $\|\cdot\|_{\alpha}$. Hence it follows from condition $(\mathbf{F})$ that $u(t) \in X_{\alpha}$ for $t \in[s, T]$ and $u(\cdot)$ is continuous over $[s, T]$ with respect to the metric topology of $X$.

It is now clear from (4.2) that the step function $u^{n}$ converges uniformly on $[s, T]$ to $u$ with respect to the metric topology of $X$. So, the lower semicontinuity of the functionals $\mathfrak{p}$ and $\mathfrak{q}_{\alpha}$ and the estimate (3.3) together yield 
and

$$
\mathfrak{p}(u(t)) \leqq \exp [a(t-s)]\left[\mathfrak{p}\left(u_{0}\right)+a^{\prime}(t-s)\right] \leqq \alpha
$$

$$
\mathfrak{q}_{\alpha}(u(t)) \leqq \exp \left[b_{\alpha}(t-s)\right]\left[\mathfrak{q}_{\alpha}\left(u_{0}\right)+b_{\alpha}^{\prime}(t-s)\right] \leqq \gamma
$$

for $t \in[s, T]$. Therefore, $u(\cdot)$ gives a weak solution of $(D E)_{s}$ constrained in $X_{\alpha}^{r}$ which satisfies $u(s)=u_{0}$.

Step 3. Let $u(\cdot)$ be the weak solution of $(D E)_{s}$ obtained by (4.3). In this step we first show that if the initial-value $u_{0}$ belongs to the set $\hat{D}(\beta ; \alpha, \delta)$ defined by (2.8) and the function $\theta_{\alpha}^{r}$ in (3.1) belongs to the class $\mathscr{C}_{B V}$ then $u(\cdot)$ is Lipschitz continuous over $[s, T]$ in the sense that the inequality

$$
\left\|u(t)-u\left(t^{\prime}\right)\right\|_{\alpha} \leqq\left|t-t^{\prime}\right| \exp [\omega(T-s)]\left[\left|A(s) u_{0}\right|_{\alpha}+3 \exp \left(\omega \lambda_{\alpha}\right) M\right]
$$

holds for $t, t^{\prime} \in[s, T]$, where $\left|A(s) u_{0}\right|_{\alpha}$ is defined by (2.7) and $M$ is a constant such that $\sum_{p=1}^{N(n)} \theta_{\alpha}^{r}\left(t_{p}^{n}, t_{p-1}^{n}\right) \leqq M$ for $n \geqq 1$.

Let $u_{0} \in \hat{D}(\beta ; \alpha, \delta)$ and define $\left\{\left(u_{k}^{n}, v_{k}^{n}\right)\right\}$ by (3.2) for a sequence of partitions $\Delta_{n}=\left\{s=t_{0}^{n}<t_{1}^{n}<\cdots<t_{N(n)}^{n}=T\right\}, n \geqq 1$, such that $\left|\Delta_{n}\right| \rightarrow 0$ as $n \rightarrow \infty$. Setting $\lambda=h_{k}^{n}, \mu=h_{k-1}^{n}, s=t_{k}^{n}, t=t_{k-1}^{n}, u=u_{k-1}^{n}$ and $v=u_{k-2}^{n}$ in Lemma 3.1, we have

$$
\left(1-\omega h_{k}^{n}\right)\left\|v_{k}^{n}\right\|_{\alpha} \leqq\left\|v_{k-1}^{n}\right\|_{\alpha}+3 \exp \left(\omega \lambda_{\alpha}\right) \theta_{\alpha}^{r}\left(t_{k}^{n}, t_{k-1}^{n}\right) \quad \text { for } 2 \leqq k \leqq N(n),
$$

where $\left|\Delta_{n}\right|<\lambda_{\alpha} / 2$. Moreover, $\left(1-\omega h_{1}^{n}\right)\left\|v_{1}^{n}\right\|_{\alpha} \leqq\left|A\left(t_{1}^{n}\right) u_{0}\right|_{\alpha} \leqq\left|A(s) u_{0}\right|_{\alpha}+\theta_{\alpha}^{r}\left(t_{1}^{n}, s\right)$. Combination of this with the above estimates gives

$$
\prod_{p=1}^{k}\left(1-\omega h_{p}^{n}\right)\left\|v_{k}^{n}\right\|_{\alpha} \leqq\left|A(s) u_{0}\right|_{\alpha}+3 \exp \left(\omega \lambda_{\alpha}\right) \sum_{p=1}^{k} \theta_{\alpha}^{r}\left(t_{p}^{n}, t_{p-1}^{n}\right)
$$

for $1 \leqq k \leqq N(n)$ and $n \geqq 1$. On the other hand, for every $n \geqq 1$ and every pair $(j, k)$ with $0 \leqq j \leqq k \leqq N(n)$, one has

$$
\left\|u_{k}^{n}-u_{j}^{n}\right\|_{\alpha} \leqq \sum_{p=j+1}^{k} h_{p}^{n}\left\|v_{p}^{n}\right\|_{\alpha}
$$

Applying (4.6) and then (4.2) to this inequality, we obtain the desired estimate (4.5).

Next, we show that if $\alpha>\beta$ then $\hat{D}\left(\beta^{\prime} ; \alpha, \delta^{\prime}\right)$ is dense in $X_{\beta} \cap X_{\alpha}^{\delta}$ provided $\delta^{\prime}>\delta$ and $\alpha>\beta^{\prime}>\beta$. Let $z \in X_{\beta} \cap X_{\alpha}^{\delta}, \delta^{\prime}>\delta, \alpha>\beta^{\prime}>\beta, r \in[0, T]$, and set $z_{\nu}=$ $J(\nu ; r) z$ for $\nu \in\left(0, \lambda_{\alpha}\right)$. Since $\mathfrak{p}\left(z_{\nu}\right) \leqq(1-a \nu)^{-1}\left[\mathfrak{p}(z)+a^{\prime} \nu\right] \leqq \beta^{\prime} \leqq \alpha$ and $\mathfrak{q}_{\alpha}\left(z_{\nu}\right) \leqq$ $\exp \left(b_{\alpha} \nu\right)\left[\mathfrak{q}_{\alpha}(z)+b_{\alpha}^{\prime} \nu\right] \leqq \delta^{\prime}$ for $\nu$ sufficiently small, we see with the aid of Lemma 3.2 that $z_{\nu} \in X_{\beta^{\prime}} \cap X_{\alpha}^{\beta^{\prime}} \cap D(A(r)) \subset \hat{D}\left(\beta^{\prime} ; \alpha, \delta^{\prime}\right)$ and $z_{\nu} \rightarrow z$ in the metric topology of $X$ as $\nu \rightarrow 0$. This means that the set $\hat{D}\left(\beta^{\prime} ; \alpha, \delta^{\prime}\right)$ is dense in $X_{\beta} \cap X_{\alpha}^{\delta}$.

Step 4. Let $s \in[0, T)$ and $\beta>0$. In this step we show that the convergence

$$
u(t, s ; v)=X-\lim \prod_{p=1}^{k}\left(I-h_{p}^{n} A\left(t_{p}^{n}\right)\right)^{-1} v
$$


is obtained for $v \in X_{\beta}$ and $t \in[s, T]$, where the limit is taken in the metric topology of $X$ as $\left|\Delta_{n}\right| \rightarrow 0$ and $t_{k}^{n} \rightarrow t$ in [s,T], and then we prove that given a pair $s, t \in[0, T]$ with $s<t$ the mapping $v \rightarrow u(t, s ; v)$ is Lipschitz continuous on $X_{\beta}$ in the sense of the norm $\|\cdot\|_{\alpha}$. Further, it will be shown during the course of the proof that (4.7) holds for any sequence $\left(\Delta_{n}\right)$ of partitions of $[s, T]$ with $\left|\Delta_{n}\right| \rightarrow 0$ and the limit $u(t, s ; v)$ does not depend upon the choice of such $\left(\Delta_{n}\right)$.

Let $s \in[0, T), \beta>0, \alpha>e^{2 a T}\left(\beta+\alpha^{\prime} T\right)$, and let $\left(A_{n}\right)$ be a sequence of partitions of $[s, T]$ with $\left|\Delta_{n}\right| \rightarrow 0$. Then conditions (A.2), (3.1) and (3.3) together imply that the family $\left\{\prod_{p=1}^{k} J\left(h_{p}^{n} ; t_{p}^{n}\right): 1 \leqq k \leqq N(n),\left|\Delta_{n}\right|<\lambda_{\alpha}\right\}$ is equi-Lipschitz continuous on $X_{\beta}$, and that a Lipschitz constant of $\prod_{p=1}^{c} J\left(h_{p}^{n} ; t_{p}^{n}\right)$ is given by $\exp \left[\omega\left(t_{k}^{n}-s\right) /\left(1-\omega\left|\Delta_{n}\right|\right)\right]$ for $1 \leqq k \leqq N(n)$ and $n$ sufficiently large. Let $v \in X_{\beta}$. Then by conditions (S.2) and (F) there is a sequence $\left(v_{l}\right)$ in $X_{\beta} \cap D\left(q_{\alpha}\right)$ such that $\left\|v-v_{l}\right\|_{\alpha} \rightarrow 0$ as $l \rightarrow \infty$. Since

$$
\left\|\prod_{p=1}^{k} J\left(h_{p}^{n} ; t_{p}^{n}\right) v-\prod_{p=1}^{k} J\left(h_{p}^{n} ; t_{p}^{n}\right) v_{l}\right\|_{\alpha} \leqq \exp \left[\omega\left(t_{k}^{n}-s\right) /\left(1-\omega\left|\Delta_{n}\right|\right)\right]\left\|v-v_{l}\right\|_{\alpha}
$$

for $1 \leqq k \leqq N(n)$ and $n$ sufficiently large, it follows from (4.2) that $\prod_{p=1}^{k} J\left(h_{p}^{n} ; t_{p}^{n}\right) v$ converges to some limit $u(t, s ; v)$ in $X$ as $n \rightarrow \infty$ and $t_{k}^{n} \rightarrow t$ in $[s, T]$ and, as mentioned at the end of Step 1 , the limit $u(t, s ; v)$ does not depend upon the choice of a sequence of partitions $\left(A_{n}\right)$. This means that for $s \in[0, T), \beta>0$ and $v \in X_{\beta}$ we get the convergence (4.7) in $X$, and that the limit function $u(\cdot, s ; v)$ gives a weak solution of $(D E)_{s}$ satisfying $u(s, s ; v)=v$.

The mapping $u(\cdot, \cdot ; v)$ which assigns to each $(t, s)$ with $0 \leqq s \leqq t \leqq T$ the limit $u(t, s ; v)$ of $(4.7)$ defines a strongly continuous function on the triangle $\{(t, s): 0 \leqq s \leqq t \leqq T\}$. In fact, let $\beta>0, \alpha>e^{2 a T}\left(\beta+a^{\prime} T\right), \delta>0$ and let $\gamma>$ $e^{b_{\alpha} T}\left(\delta+b_{\alpha}^{\prime} T\right)$, where $a, a^{\prime}, b_{\alpha}, b_{\alpha}^{\prime}$ are constants specified by (S.1) and (S.2). Let $s, \hat{s} \in[0, T)$ and $v \in X_{\beta} \cap X_{\alpha}^{\delta}$. We then take any sequence $\left(\Delta_{n}\right)$ of partitions of $[s, T]$ with $\left|\Delta_{n}\right| \rightarrow 0$ and any sequence $\left(\hat{\Delta}_{m}\right)$ of partitions of $[\hat{s}, T]$ with $\left|\hat{\Delta}_{m}\right| \rightarrow 0$ and define two sequences $\left(u_{k}^{n}\right)$ and $\left(\hat{u}_{j}^{m}\right)$ by (3.4). Let $t \in[s, T], \hat{t} \in[\hat{s}, T], \eta=t$ $-\hat{t},|t-\hat{t}|<\xi \leqq T, 0<\kappa<\xi-|t-\hat{t}|, z=\hat{u}_{0}=u_{0}=v$ and $z_{\nu}=J(\nu ; r) v$ for $\nu \in\left(0, \lambda_{\alpha} / 2\right)$ in (3.9). Taking the limit in the resultant inequality as $t_{k}^{n} \rightarrow t$ and $\hat{t}_{j}^{m} \rightarrow \hat{t}$, we see that

$$
\begin{aligned}
& \exp [-\omega(t+\hat{t}-s-\hat{s})]\|u(t, s ; v)-u(\hat{t}, \hat{s} ; v)\|_{\alpha} \\
& \quad \leqq 2\left\|v-z_{\nu}\right\|_{\alpha}+(|t-\hat{t}|+|s-\hat{s}|)\left[\left\|w_{\nu}\right\|_{\alpha}+3 e^{\omega \lambda \alpha} \rho_{\alpha}^{\gamma}(T)\right]+3 e^{\omega \lambda \alpha}(\hat{t}-\hat{s}) \rho_{\alpha}^{\gamma}(\xi)
\end{aligned}
$$

holds for $\nu \in\left(0, \lambda_{\alpha} / 2\right)$, where $z_{\nu}=J(\nu ; r) v$. Hence, passing to the limit as $\xi \downarrow|t-\hat{t}|$, one obtains

$$
\begin{aligned}
& \exp (-2 \omega T)\|u(t, s ; v)-u(\hat{t}, \hat{s} ; v)\|_{\alpha} \\
& \quad \leqq 2\left\|v-z_{\nu}\right\|_{\alpha}+(|t-\hat{t}|+|s-\hat{s}|)\left(\left\|w_{\nu}\right\|_{\alpha}+3 e^{\omega \lambda \alpha} \rho_{\alpha}^{\tau}(T)\right)+3 e^{\omega \lambda \alpha}(\hat{t}-\hat{s}) \rho_{\alpha}^{\tau}(|t-\hat{t}|+0) .
\end{aligned}
$$


This means that the mapping $(t, s) \rightarrow u(t, s ; v)$ is strongly continuous over the triangle $0 \leqq s \leqq t \leqq T$. Next let $v$ be an arbitrary element of $X_{\beta}$. Then, by conditions (S.2) and (F), one finds a sequence $\left\{v_{l}\right\}$ in $X_{\beta} \cap D\left(q_{\alpha}\right)$ such that $\left\|v-v_{l}\right\|_{\alpha} \rightarrow 0$ as $l \rightarrow \infty$. Therefore, from (4.7), (4.8) and the estimates

$$
\begin{aligned}
& \|u(t, s ; v)-u(\hat{t}, \hat{s} ; v)\|_{\alpha} \\
& \quad \leq 2 \exp (\omega T)\left\|v-v_{l}\right\|_{\alpha}+\left\|u\left(t, s ; v_{l}\right)-u\left(\hat{t}, \hat{s} ; v_{l}\right)\right\|_{\alpha}, \quad l \geqq 1,
\end{aligned}
$$

it follows that $u(t, s, v)$ is strongly continuous over the triangle $0 \leqq s \leqq t \leqq T$.

Finally, let $\alpha, \beta$ and $\left(\Delta_{n}\right)$ be as specified above and take any pair $v, \hat{v}$ in $X_{\beta}$. Then there eixst two $X$-valued, strongly continuous functions $u(t, s ; v)$ and $u(t, s ; \hat{v})$ over the triangle $0 \leqq s \leqq t \leqq T$. Now (3.1), (3.3), and (4.7) together imply that

$$
\|u(t, s ; v)-u(t, s ; \hat{v})\|_{\alpha} \leqq \exp [\omega(t-s)]\|v-\hat{v}\|_{\alpha}
$$

for $0 \leqq s \leqq t \leqq T$. This means that the limit function $u(t, s ; v)$ obtained by (4.7) depends continuously upon the initial-value $v$ in $X_{\beta}$ in the sense of the metric topology of $X$.

Step 5. Let $\beta>0$ and $\alpha^{\prime} \geqq \alpha>\exp (2 a T)\left(\beta+a^{\prime} T\right)$. Let $v \in X_{\beta}, s \in[0, T)$, and let $u(\cdot) \equiv u(\cdot, s ; v)$ be the associated weak solution of $(D E)_{s}$ obtained by (4.7). In this step we demonstrate that $u(\cdot)$ is the only weak solution of $(D E)_{s}$ constrained in $X_{\alpha^{\prime}}$ which satisfies $u(s)=v$. Let $\tilde{u}:[s, T] \rightarrow X$ be any weak solution with initial-value $v$ of $(D E)_{s}$ constrained in $X_{\alpha^{\prime}}$, and let $\left(\Delta_{n}, \tilde{u}_{n}, \tilde{f}_{n}\right)$ be the associated sequence of partitions, approximate solutions and error functions as mentioned in conditions $\left(\mathbf{w}_{1}\right)$ through $\left(\mathbf{w}_{3}\right)$ with $C=X_{\alpha^{\prime}}$, where we write $\Delta_{n}=\left\{s=t_{0}^{n}<t_{1}^{n}<\cdots<t_{N(n)}^{n}=T\right\}, h_{k}^{n}=t_{k}^{n}-t_{k-1}^{n}, \tilde{u}_{k}^{n}=\tilde{u}_{n}\left(t_{k}^{n}\right)$, and $\tilde{f}_{n}=\sum_{k-1}^{N(n)} \tilde{z}_{k}^{n} \chi_{\left(t_{k-1}^{n}, t_{k}^{n}\right]}$ for $1 \leqq k \leqq N(n)$. Then condition $\left(\mathbf{w}_{1}\right)$ states that $\left(h_{k}^{n}\right)^{-1} \times$ $\left[\tilde{u}_{k}^{n}-\tilde{u}_{k-1}^{n}\right]=\tilde{v}_{k}^{n}+\tilde{z}_{k}^{n}$ and $\left(\tilde{u}_{k}^{n}, \tilde{v}_{k}^{n}\right) \in A\left(t_{k}^{n}\right)$ and $\tilde{u}_{k}^{n} \in X_{\alpha^{\prime}}$, and hence $\tilde{u}_{k-1}^{n}+h_{k}^{n} \tilde{z}_{k}^{n} \in$ $\left(I-h_{k}^{n} A\left(t_{k}^{n}\right)\right) \tilde{u}_{k}^{n} \subset X_{0}$ for $1 \leqq k \leqq N(n)$ and $n \geqq 1$. So, if $\left|\Delta_{n}\right|<\lambda_{\alpha^{\prime}}\left(\lambda_{\alpha^{\prime}}\right.$ being a positive constant furnished by condition (S.2)), then $\tilde{u}_{k}^{n}=J\left(h_{k}^{n} ; t_{k}^{n}\right)\left(\tilde{u}_{k-1}^{n}+h_{k}^{n} \tilde{z}_{k}^{n}\right) \in X_{\alpha^{\prime}}$ and $\tilde{v}_{k}^{n}=A\left(t_{k}^{n} ; h_{k}^{n}\right)\left(\tilde{u}_{k-1}^{n}+h_{k}^{n} \tilde{z}_{k}^{n}\right)$ for $1 \leqq k \leqq N(n)$ and $n \geqq 1$. On the other hand, as shown in the preceding steps, $u_{k}^{n}$ obtained for the same sequence $\left(\Delta_{n}\right)$ of partitions as above by (3.2) converges to the weak solution $u$ in the sense that $\left\|u_{k}^{n}-u(t)\right\|_{\alpha} \rightarrow 0$ and therefore $\left\|u_{k}^{n}-u(t)\right\|_{\alpha^{\prime}} \rightarrow 0$ as $n \rightarrow \infty$ and $t_{k}^{n} \rightarrow t$ by (F).

Now the application of (A.2) yields

$$
\begin{aligned}
\left\|u_{k}^{n}-\tilde{u}_{k}^{n}\right\|_{\alpha^{\prime}} & =\left\|J\left(h_{k}^{n} ; t_{k}^{n}\right) u_{k-1}^{n}-J\left(h_{k}^{n} ; t_{k}^{n}\right)\left(\tilde{u}_{k-1}^{n}+h_{k}^{n} \tilde{z}_{k}^{n}\right)\right\|_{\alpha^{\prime}} \\
& \leqq\left(1-\omega h_{k}^{n}\right)^{-1}\left[\left\|u_{k-1}^{n}-\tilde{u}_{k-1}^{n}\right\|_{\alpha^{\prime}}+h_{k}^{n}\left\|\tilde{z}_{k}^{n}\right\|_{\alpha^{\prime}}\right] \\
& \leqq \prod_{j=1}^{k}\left(1-\omega h_{j}^{n}\right)^{-1}\left[\sum_{j=1}^{k} h_{j}^{n}\left\|\tilde{z}_{j}^{n}\right\|_{\alpha^{\prime}}\right] \\
& \leqq(T-s) \sum_{j=1}^{N(n)}\left(1-\omega h_{j}^{n}\right)^{-1} \sup \left\{\left\|\tilde{z}_{j}^{n}\right\|_{\alpha^{\prime}}: 1 \leqq j \leqq N(n)\right\}
\end{aligned}
$$


But conditions $\left(\mathbf{w}_{2}\right)$ and $(\mathbf{F})$ together imply that the right side goes to 0 as $n \rightarrow \infty$, and so it follows from conditions $\left(\mathbf{w}_{3}\right)$ and $(\mathbf{F})$ that $\left\|u_{k}^{n}-\tilde{u}(t)\right\|_{\alpha^{\prime}} \rightarrow 0$ as $n \rightarrow \infty$ and $t_{k}^{n} \rightarrow t$. This means that $\|\tilde{u}(t)-u(t)\|_{\alpha^{\prime}} \equiv 0$ on $[s, T]$. Thus $u(\cdot)$ is the unique weak solution of $(D E)_{s}$ constrained in $X_{\alpha^{\prime}}$ with $u(s)=v$.

Step 6. Here we construct an evolution operator $\mathscr{U}$ on $X_{0}$ as stated in Theorem 1. Given $s \in[0, T), t \in[s, T]$ and $v \in X_{\beta}$, we define

$$
U(t, s) v=u(t, s ; v),
$$

where $u(t, s ; v)$ is the limit obtained for $(t, s, v)$ by (4.7). Then $U(t, s) v \in X_{\alpha}$ for $\alpha \geqq \exp (2 a T)\left(\beta+a^{\prime} Y\right)$ and the function $U(\cdot, s) v$ gives a unique weak solution with initial-value $v$ of $(D E)_{s}$ and constrained in $X_{\alpha^{\prime}}$ for $\alpha^{\prime} \geqq \alpha$, Since $v$ is arbitrary in $X_{0}$, one can define single-valued operators $U(t, s), 0 \leqq s \leqq t \leqq T$, on $X_{0}$ into itself such that for every $v \in X_{0}, U(t, s) v$ is strongly continuous over the triangle $0 \leqq s \leqq t \leqq T$. Moreover, we infer from the unicity of weak solutions established in Step 5 that $U(t, r)=U(t, s) U(s, r)$ on $X_{0}$ for $0 \leqq r \leqq s \leqq$ $t \leqq T$. Thus one obtains an evolution operator $\mathscr{U}=\{U(t, s): 0 \leqq s \leqq t \leqq T\}$ on $X_{0}$. It is now clear from the definition (4.9) of $\mathscr{U}$ and the properties of the limit functions $u(t, s ; v)$ (which have been discussed in the preceding steps) that $\mathscr{U}$ has properties $(a)$ through $(f)$ listed in the statement of Theorem 1.

\section{§. A generalization}

In this section we give a generalization of Theorem 1 . It is possible to construct an evolution operator on $X_{0}$ under weaker conditions that allow the constants $\omega$ and $\lambda_{0}$ to depend upon $\alpha$. In what follows, we denote by $A_{\alpha}(t)$ the restriction of $A(t)$ to $X_{\alpha}$ for each $\alpha>0$, namely:

$$
A_{\alpha}(t)=A(t)_{X_{\alpha}}=\left\{(u, v) \in X \times X: u \in X_{\alpha}, v \in A(t) u\right\} .
$$

In order that the same method as in the preceding section can be applied to get the evolution operator on $X_{0}$, it is sufficient to assume that the family $\{A(t): t \in[0, T]\}$ satisfies the following conditions:

$\left(\mathbf{A}^{\prime} .1\right)$ For every $t \in\left[0, T^{\prime}\right]$ and every $\alpha>0$, the domain $D\left(A_{\alpha}(t)\right)$ is dense in $X_{\alpha}$ and $R\left(A_{\alpha}(t)\right) \subset X_{0}$.

$\left(\mathbf{A}^{\prime} .2\right)$ For every $\alpha>0$, there exist constants $\omega_{\alpha} \geqq 0$ and $\lambda_{\alpha} \in\left(0,1 / \omega_{\alpha}\right)$ such that the inequality

$$
\left(1-\omega_{\alpha} \lambda\right)\|v-w\|_{\alpha} \leqq\left\|\left(v-\lambda v^{\prime}\right)-\left(w-\lambda w^{\prime}\right)\right\|_{\alpha}
$$

holds for $v, w \in D\left(A_{\alpha}(t)\right), v^{\prime} \in A_{\alpha}(t) v, w^{\prime} \in A_{\alpha}(t) w, \lambda \in\left(0, \lambda_{\alpha}\right)$ and $t \in[0, T]$.

$\left(\mathbf{A}^{\prime} .3\right)$ There exists a nondecreasing function $f:(0, \infty) \rightarrow(0, \infty)$ such that $f(\beta) \geqq \beta$ on $(0, \infty)$ and if $\beta>0$ and $\alpha>f(\beta)$, then we have 


$$
R\left(I-\lambda A_{\alpha}(t)\right) \supset X_{\beta}
$$

for $\lambda \in\left(0, \lambda_{\alpha}\right)$ and $t \in[0, T]$.

Conditions $\left(\mathbf{A}^{\prime} . \mathbf{1}\right),\left(\mathbf{A}^{\prime} .2\right)$ and $\left(\mathbf{A}^{\prime} .3\right)$ together imply that for $\beta>0, \alpha>f(\beta)$, $\lambda \in\left(0, \lambda_{\alpha}\right)$ and $t \in[0, T]$, the operator $I-\lambda A_{\alpha}(t)$ is injective and the resolvent of $A_{\alpha}(t)$

$$
J_{\alpha}(\lambda ; t)=\left(I-\lambda A_{\alpha}(t)\right)^{-1}
$$

exists as a single-valued operator with domain containing $X_{\beta}$, and that

$$
\left\|J_{\alpha}(\lambda ; s) v-J_{\alpha}(\lambda ; t) w\right\|_{\alpha} \leqq\left(1-\omega_{\alpha} \lambda\right)^{-1}\|v-w\|_{\alpha}
$$

for $v, w \in X_{\beta}$. Conditions $\left(\mathbf{A}^{\prime} .2\right)$ and $\left(\mathbf{A}^{\prime} .3\right)$ are therefore localized forms of (A.2) and (A.3), respectively. Notice that $\left(\mathbf{A}^{\prime} . \mathbf{1}\right)$ is equivalent to (A.1).

We then put the following conditions which are parallel to $(\mathbf{S . 1}),(\mathbf{S . 2})$ and (S.3), respectively.

$\left(\mathbf{S}^{\prime} . \mathbf{1}\right)$ There exist constants $a, \alpha^{\prime} \geqq 0$ such that if $\beta>0$ and $\alpha>f(\beta)$, then we have

$$
\mathfrak{p}\left(J_{\alpha}(\lambda ; t) v\right) \leqq(1-a \lambda)^{-1}\left[\mathfrak{p}(v)+a^{\prime} \lambda\right]
$$

for $\lambda \in\left(0, \lambda_{\alpha}\right)$ and $v \in X_{\beta}$.

$\left(\mathbf{S}^{\prime} .2\right)$ For every $\alpha>0$ there exists an 1.s.c, convex functional $\mathfrak{q}_{\alpha}: X \rightarrow$ $[0, \infty]$ and constants $b_{\alpha}, b_{\alpha}^{\prime} \geqq 0$ such that $D\left(\mathfrak{q}_{\alpha}\right)$ is dense in $X_{\beta}$ for $\beta \in(0, \alpha]$ and the inequality

$$
\mathfrak{q}_{\alpha}\left(J_{\alpha}(\lambda ; t) v\right) \leqq \exp \left(b_{\alpha} \lambda\right)\left[\mathfrak{q}_{\alpha}(v)+b_{\alpha}^{\prime} \lambda\right]
$$

holds for $\lambda \in\left(0, \lambda_{\alpha}\right), t \in[0, T]$ and $v \in X_{\alpha} \cap D\left(\mathfrak{q}_{\alpha}\right)$ satisfying $f(\beta(v, \lambda))<\alpha$ with $\beta(v, \lambda)=(1-a \lambda)^{-1}\left[\mathrm{p}(v)+a^{\prime} \lambda\right]$.

$\left(\mathbf{S}^{\prime} .3\right)$ For $\alpha>0$ and $\gamma>0$ there exists a function $\theta_{\alpha}^{r}$ of class $\mathscr{C}$ such that the inequality

$$
\left(1-\omega_{\alpha} \lambda\right)\left\|J_{\alpha}(\lambda ; t) v-J_{\alpha}(\lambda ; s) v\right\|_{\alpha} \leqq \lambda \theta_{\alpha}^{\gamma}(t, s)
$$

holds for $s, t \in[0, T], \lambda \in\left(0, \lambda_{\alpha}\right)$ and $v \in X$ satisfying

$$
f\left((1-a \lambda)^{-1}\left[\mathfrak{p}(v)+a^{\prime} \lambda\right]\right)<\alpha \text { and } \exp \left(b_{\alpha} \lambda\right)\left[\mathfrak{q}_{\alpha}(v)+b_{\alpha}^{\prime} \lambda\right]<\gamma .
$$

Under these conditions, we obtain the following result which gives a generalization of Theorem 1.

TheOREM 2. Under conditions $(\mathbf{F}),\left(\mathbf{A}^{\prime} . \mathbf{i}\right)$ and $\left(\mathbf{S}^{\prime} . \mathbf{i}\right), i=1,2,3$, there exists an evolution operator $\mathscr{U}$ on $X_{0}$ with the following properties:

(a) Let $s \in[0, T), \beta>0, \beta^{\prime} \geqq e^{2 a T}\left(\beta+a^{\prime} T\right)$ and $\alpha>f\left(\beta^{\prime}\right)$. Then for every sequence $\left(\Delta_{n}\right)$ of partitions of $[s, T]$ with $\left|\Delta_{n}\right| \rightarrow 0$, we have the convergence 


$$
U(t, s) v=\lim \prod_{p=1}^{k} J_{\alpha}\left(h_{p}^{n} ; t_{p}^{n}\right) v \quad \text { for } \quad t \in[s, T] \text { and } v \in X_{\beta}
$$

where the limit is taken as $n \rightarrow \infty$ and $t_{k}^{n} \rightarrow t$ in $[s, T]$.

$(\mathrm{b})^{\prime} \quad$ Let $s \in[0, T), \beta>0, \beta^{\prime} \geqq e^{2 a T}\left(\beta+\alpha^{\prime} T\right), \alpha>f\left(\beta^{\prime}\right)$, and let $v \in X_{\beta}$. Then the function $u(t) \equiv U(t, s) v$ gives a unique weak solution of $(D E)_{s}$ constrained in $X_{\alpha}$ satisfying $u(s)=v$.

(c) Given $s, t \in[0, T]$ with $s \leqq t, \beta>0, \beta^{\prime} \geqq e^{2 a T}\left(\beta+a^{\prime} T\right)$, and $\alpha>f\left(\beta^{\prime}\right)$, $U(t, s)$ maps $X_{\beta}$ into $X_{\alpha}$ and we have

$$
\|U(t, s) v-U(t, s) w\|_{\alpha} \leqq \exp \left[\omega_{\alpha}(t-s)\right]\|v-w\|_{\alpha}
$$

for $v, w \in X_{\beta}$ and $0 \leqq s \leqq t \leqq T$. Therefore, for each $\beta>0$, the family of operators $\{U(t, s)\}$ is equicontinuous on $X_{\beta}$ with respect to the topology of $X$.

$(\mathrm{d})^{\prime} \quad \mathfrak{p}(U(t, s) v) \leqq e^{a(t-s)}\left[\mathfrak{p}(v)+a^{\prime}(t-s)\right] \quad$ for $v \in X_{0}$ and $0 \leqq s \leqq t \leqq T$.

(e) Let $\beta, \delta>0, \beta^{\prime} \geqq e^{2 a T}\left(\beta+a^{\prime} T\right), \alpha>f\left(\beta^{\prime}\right)$, and $\gamma>e^{b_{\alpha} T}\left(\delta+b_{\alpha}^{\prime} T\right)$. Then

$$
\mathfrak{q}_{\alpha}(U(t, s) v) \leqq \exp \left[b_{\alpha}(t-s)\right]\left[\mathfrak{q}_{\alpha}(v)+b_{\alpha}^{\prime}(t-s)\right] \leqq \gamma
$$

for $v \in X_{\beta} \cap X_{\alpha}^{\delta}$ and $0 \leqq s \leqq t \leqq T$.

$(\mathrm{f})^{\prime} \quad$ Let $\beta>0, \delta>0, \beta^{\prime} \geqq e^{2 a T}\left(\beta+a^{\prime} T\right), \alpha>f\left(\beta^{\prime}\right)$, and let $\gamma>e^{b_{\alpha} T}\left(\delta+b_{\alpha}^{\prime} T\right)$. Then the limit $|A(t) v|_{\alpha}=\lim _{\lambda \rightarrow 0}\left\|A_{\alpha}(t ; \lambda) v\right\|_{\alpha}$ exists for $v \in X_{\beta} \cap X_{\alpha}^{\delta} . \quad$ Let $\hat{D}(\beta ; \alpha, \gamma)$ be defined by (2.8). Then the set $\hat{D}\left(\beta^{\prime} ; \alpha, \delta^{\prime}\right)$ is dense in $X_{\beta} \cap X_{\alpha}^{\delta}$ provided that $\delta^{\prime}>\delta, \beta^{\prime}>\beta$ and $\alpha>f\left(\beta^{\prime}\right)$. If $v \in \hat{D}(\beta ; \alpha, \delta), \theta_{\alpha}^{r}$ is the function specified by $\left(\mathbf{S}^{\prime} .3\right)$ and $\theta_{\alpha}^{r}$ belongs to the class $\mathscr{C}_{B V}$, then the weak solution $u(t) \equiv U(t, s) v$ with initial-value $v$ of $(D E)_{s}$ is Lipschitz continuous over $[s, T]$ with respect to the norm $\|\cdot\|_{\alpha}$.

The above theorem is verified through a modification of the proof of Theorem 1. We give here a brief exposition as to how we follow the arguments of Sections 3 and 4 under the assumptions of Theorem 2.

First we observe that under conditions $(\mathbf{F}),\left(\mathbf{A}^{\prime} . \mathbf{i}\right)$ and $\left(\mathbf{S}^{\prime} \mathbf{. 1}\right)$ an approximating discrete scheme for the system $\left\{(D E)_{s}\right\}$ can be constructed in a way similar to the case of Theorem 1. Let $s \in[0, T), \beta>0, \beta^{\prime} \geqq e^{2 a T}\left(\beta+a^{\prime} T\right)$, and let $\alpha>f\left(\beta^{\prime}\right)$. Then for every sequence $\left(\Delta_{n}\right)$ of partitions of $[s, T]$ such that $\Delta_{n}=\left\{s=t_{0}^{n}<t_{1}^{n}<\cdots<t_{N(n)}^{n}=T\right\}$ and $\left|\Delta_{n}\right|<\lambda_{\alpha}$ for $n \geqq 1$, we infer from (A'.3) that $R\left(I-\lambda A_{\alpha}(t)\right) \supset X_{\beta(n, k)}$ for $\beta(n, k)=\prod_{p=1}^{k}\left(1-a h_{p}^{n}\right)^{-1}\left(\beta+\left(t_{k}^{n}-s\right) a^{\prime}\right), h_{p}^{n}=t_{p}^{n}-t_{p-1}^{n}, 1 \leqq$ $\leqq p \leqq k \leqq N(n)$ and $n \geqq 1$. Therefore the aimed discrete shceme $\left\{\left(u_{k}^{n}, v_{k}^{n}\right): 1 \leqq k\right.$ $\leqq N(n), n \geqq 1\}$ is obtained through (2.4) with $J\left(h_{p}^{n} ; t_{p}^{n}\right)$ and $A\left(t_{k}^{n} ; h_{k}^{n}\right)$ replaced respectively by $J_{\alpha}\left(h_{p}^{n} ; t_{p}^{n}\right)$ and $A_{\alpha}\left(t_{k}^{n} ; h_{k}^{n}\right)$, where $A_{\alpha}(t ; \lambda)$ denotes the Yosida approximation of $A_{\alpha}(t)$,

$$
A_{\alpha}(t ; \lambda)=\lambda^{-1}\left[J_{\alpha}(\lambda ; t)-I\right], \quad \lambda \in\left(0, \lambda_{a}\right) .
$$


Thus, the evolution operator $\mathscr{U}$ stated in Theorem 2 can be obtained by showing the convergence (2.5).

Secondly, the only difference between (S.2) and $\left(\mathbf{S}^{\prime} .2\right)$ is that $J(\lambda ; t) v$ is replaced in $\left(\mathbf{S}^{\prime} .2\right)$ by $J_{\alpha}(\lambda ; t) v$; hence we can define closed convex subsets $X_{\alpha}^{r}$, $\alpha>0, \gamma>0$, by (2.6). Let $\beta>0, \delta>0$ and $\alpha>f(\beta)$. Then, for every $v \in X_{\beta} \cap X_{\alpha}^{\delta}$, the function $\lambda \rightarrow\left(1-\lambda \omega_{\alpha}\right)\left\|A_{\alpha}(t ; \lambda) v\right\|_{\alpha}$ is monotone nonicreasing on $\left(0, \lambda_{\alpha}\right)$ by conditions ( $\left.\mathbf{A}^{\prime} .2\right)$ and $\left(\mathbf{A}^{\prime} .3\right)$, and so we can define the functional $v \rightarrow|A(t) v|_{\alpha}$ on $X_{\beta} \cap X_{\alpha}^{\delta}$ through $(2.7)$ with $A(t ; \lambda)$ replaced by $A_{\alpha}(t ; \lambda)$. In view of this the set $\hat{D}(\beta ; \alpha, \delta)$ is defined by (2.8). Moreover conditions $\left(\mathbf{A}^{\prime} . \mathbf{2}\right)$ and $\left(\mathbf{S}^{\prime} . \mathbf{3}\right)$ together yield

$$
\left(1-\omega_{\alpha} \lambda\right)\left\|J_{\alpha}(\lambda ; s) v-J_{\alpha}(\lambda ; t) w\right\|_{\alpha} \leqq\|v-w\|_{\alpha}+\lambda \theta_{\alpha}^{\gamma}(s, t)
$$

for $s, t \in[0, T], \lambda \in\left(0, \lambda_{\alpha}\right)$ and $v, w$ in $X_{\alpha}^{r}$ satisfying (5.4).

Therefore all of the basic estimates established under conditions (A.i) and $(\mathbf{S . i})$ can be extended to the case where $\left(\mathbf{A}^{\prime} \cdot \mathbf{i}\right)$ and $\left(\mathbf{S}^{\prime} \cdot \mathbf{i}\right)$ hold. First the conclusion of Lemma 3.1 is valid under $\left(\mathbf{A}^{\prime} .2\right)$ and $\left(\mathbf{S}^{\prime} .2\right)$ if $\omega, J(\lambda ; s)$ and $J(\lambda ; t)$ are replaced respectively by $\omega_{\alpha}, J_{\alpha}(\lambda ; s)$ and $J_{\alpha}(\lambda ; t)$, and if the elements $u, v$ are supposed to satisfy (5.4). Likewise, we obtain the same conclusion as in Lemma 3.2 if $\alpha$ is chosen so that $\alpha>f(\beta)$, and $\omega$ is replaced by $\omega_{\alpha}$ as stated in $\left(\mathbf{A}^{\prime} .2\right)$, and then replace $J(\nu ; r) z$ by $J_{\alpha}(\nu ; r) z$. Next let $s \in[0, T), \beta>0, \delta>0$, $\beta^{\prime} \geqq \exp (2 a T)(\beta+a T), \alpha>f\left(\beta^{\prime}\right)$, and $\gamma \geqq \exp \left(b_{\alpha} T\right)\left(\delta+b_{\alpha}^{\prime} T\right)$, where $f$ is the function as stated in $\left(\mathbf{A}^{\prime} .2\right)$ and $a, a^{\prime}, b_{\alpha}, b_{\alpha}^{\prime}$ are constants provided by $\left(\mathbf{S}^{\prime} . \mathbf{1}\right)$ and $\left(\mathbf{S}^{\prime} .2\right)$. Let $u_{0} \in X_{\beta}^{o}$ and $\left(\Delta_{n}\right)$ a sequence of partitions of $[s, T]$ such that $\left|\Delta_{n}\right|<$ $\lambda_{\alpha} / 2$ and $\left|\Delta_{n}\right| \rightarrow 0$. Then a sequence $\left(\left(u_{k}^{n}, v_{k}^{n}\right): 1 \leqq k \leqq N(n)\right)$ can be defined through (3.2) with $J\left(h_{p}^{n} ; t_{p}^{n}\right) u_{0}$ replaced by $J_{\alpha}\left(h_{p}^{n} ; t_{p}^{n}\right) u_{0}$, and (3.3) holds for the sequence by $\left(\mathbf{S}^{\prime} \cdot \mathbf{1}\right)$ and $\left(\mathbf{S}^{\prime} \cdot \mathbf{2}\right)$. Consequently, we see in the same way as in the proof of Lemma 3.3 that the conclusion of Lemma 3 remains valid if $\omega$ is replaced by $\omega_{\alpha}$ and if $\omega_{\alpha}, \lambda_{\alpha}$ are understood to be the constants given by $\left(\mathbf{A}^{\prime}\right.$.2).

Proof of Theorem 2. Using the above-mentioned basic estimates in the case where conditions $\left(\mathbf{A}^{\prime} \cdot \mathbf{1}\right)$ and $\left(\mathbf{S}^{\prime} . \mathbf{i}\right), i=1,2,3$, hold, we follow each step of the argument of Section 4. Let $s \in[0, T), \beta>0, \delta>0, \beta^{\prime} \geqq \exp (2 a T)\left(\beta+a^{\prime} T\right)$, $\alpha>f\left(\beta^{\prime}\right)$ and let $\gamma \geqq \exp \left(b_{\alpha} T\right)\left(\delta+b_{\alpha}^{\prime} T\right)$. First even though conditions (A.i) and (S.i) are replaced respectively by $\left(\mathbf{A}^{\prime} . \mathbf{i}\right)$ and $\left(\mathbf{S}^{\prime} . \mathbf{i}\right)$, we can proceed with the same argument as in Step 1 by replacing the constant $\omega$ by $\omega_{\alpha}$. Hence, given an intial-value $u_{0} \in X_{\beta} \cap X_{\alpha}^{o}$, there is a $Y$-valued function $w(\cdot)$ on $[s, T]$ such that $w(s)=u_{0}$ and $\left\|p_{\alpha} u_{k}^{n}-w(t)\right\|_{Y} \rightarrow 0$ as $n \rightarrow \infty$ and $t_{k}^{n} \rightarrow t$ in $[s, T]$. Next, replacing $\omega$ by $\omega_{\alpha}$, we see in the same way as in Steps 2 and 3 that the $X$-valued function $u(\cdot) \equiv P_{\alpha}^{-1} w(\cdot)$ gives a weak solution of $(D E)_{s}$ constrained in $X_{\alpha}$ which satisfies $u(s)=u_{0}$, and that if in particular $u_{0} \in \hat{D}(\beta ; \alpha, \delta)$ and the func- 
tion $\theta_{\alpha}^{r}$ provided by $\left(\mathbf{S}^{\prime} \cdot \mathbf{3}\right)$ belongs to the class $\mathscr{C}_{B V}$ then (4.5) holds with $\omega$ replaced by $\omega_{\alpha}$. Moreover, if $\beta_{1}>\beta, \alpha>f\left(\beta_{1}\right)$ and $\delta_{1}>\delta$, then it is shown in the same way as in the last-half of Step 3 (where it is sufficient to replace $J(\nu ; r)$ by $\left.J_{\alpha}(\nu ; r)\right)$ that $\hat{D}\left(\beta_{1} ; \alpha, \delta_{1}\right)$ is dense in $X_{\beta} \cap X_{\alpha}^{\delta}$. From this Assertion $(f)^{\prime}$ is obtained.

Let $\left(\Delta_{n}\right)$ be any sequence of partitions of $[s, T]$ with $\left|\Delta_{n}\right| \rightarrow 0$. Then applying $\left(\mathbf{A}^{\prime} . \mathbf{2}\right),\left(\mathbf{A}^{\prime} . \mathbf{3}\right),\left(\mathbf{S}^{\prime} . \mathbf{1}\right)$ and the estimate corresponding to (3.3) and using the same reasoning as in Step 4 , we see that if $1 \leqq k \leqq N(n)$ and $\left|\Delta_{n}\right|<\lambda_{\alpha}$ then $\prod_{p=1}^{k} J_{\alpha}\left(h_{p}^{n} ; t_{p}^{n}\right)$ makes sense as a Lipschitzian operator with domain containing $X_{\beta}$ and the Lipschitz constant is given by $\left.\exp \left[\omega_{\alpha}\left(t_{k}^{n}-s\right) /\left(1-\omega_{\alpha} \mid \Delta_{n}\right)\right)\right]$. From this and the same argument as in Step 4 it follows that

$$
u(t, s ; v)=X-\lim \prod_{p=1}^{k} J_{\alpha}\left(h_{p}^{n} ; t_{p}^{n}\right) v
$$

is obtained for every $v \in X_{\beta}$ and every $t \in[s, T]$, where the limit is taken in the metric topology of $X$ as $n \rightarrow \infty$ and $t_{k}^{n} \rightarrow t$ in $[s, T]$. It is shown during the course of the proof of (5.6) that the limit $u(t, s ; v)$ does not depend upon the choice of a sequence $\left(\Delta_{n}\right)$ so far as $\left|\Delta_{n}\right| \rightarrow 0$, and that given a pair $s, t \in[0, T]$ with $s \leqq t$ we have the inequality

$$
\|u(t, s ; v)-u(t, s ; \hat{v})\|_{\alpha} \leqq \exp \left[\omega_{\alpha}(t-s)\right]\|v-\hat{v}\|_{\alpha}
$$

for $v, \hat{v} \in X_{\beta}$. Further it is proved that for every $v \in X_{\beta}$ the function $u(t, s ; v)$ is strongly continuous over the triangle $0 \leqq s \leqq t \leqq T$. The last two consequences together show that $u(t, s ; v)$ depends continuously upon the initialtime $s \in[0, T)$ as well as the initial-value $v \in X_{\beta}$ in the sense of the metric topology of $X$, and thus Assertion (c) follows.

Let $\alpha^{\prime} \geqq \alpha, v \in X_{\beta}$, and let $\tilde{u}:[s, T] \rightarrow X$ be any weak solution of $(D E)_{s}$ constrained in $X_{\alpha^{\prime}}$ which satisfies $\tilde{u}(s)=v$. Let $u(\cdot, s ; v)$ be the function obtained by (5.6). Then we can derive the identity $\tilde{u}(t) \equiv u(t, s ; v)$ for $t \in[s, T]$ by replacing in Section 4, Step 5 the constant $\omega$ and the operators $A\left(t_{k}^{n}\right)$ and $J\left(h_{k}^{n} ; t_{k}^{n}\right)$ with $\omega_{\alpha^{\prime}}, A_{a^{\prime}}\left(t_{k}^{n}\right)$ and $J_{\alpha^{\prime}}\left(h_{k}^{n} ; t_{k}^{n}\right)$, respectively. This means that $u(\cdot, s ; v)$ is the only weak solution with initial value $v$ of $(D E)_{s}$ constrained in $X_{\alpha^{\prime}}$, and Assertion $(b)^{\prime}$ is obtained. Assertions $(d)^{\prime}$ and $(e)^{\prime}$ now follow from the convergence (5.6) and the estimate corresponding to (3.3),

Finally, the desired evolution operator $\mathscr{U}$ on $X_{0}$ is obtained in the same way as in Section 4, Step 6.

q.e.d.

\section{§6. Application to first-order quasilinear equations}

In this section we give two examples of evolution operators in Fréchet spaces in order to illustrate our results. The evolution operators discussed 
below are both typical examples of evolution operators of our interest, though in each case the construction of the nonlinear operators $A(t), t \in[0, T]$, is considerably complicated. Hence we here cite two results obtained in the recent work of the authors [5] and the forthcoming paper [6].

Consider the first-order quasilinear equation

$$
u_{t}+\sum_{i=1}^{d}\left(\phi_{i}(t, x, u)\right)_{x_{i}}+\psi(t, x, u)=0, \quad 0<t<T, \quad x=\left(x_{1}, \cdots, x_{d}\right) \in \boldsymbol{R}^{d}
$$

under the initial condition

$$
L_{l o c}^{1}-\lim _{t \downarrow 0} u(t, \cdot)=v
$$

where $\phi$ and $\psi$ are given nonlinear functions over $[0, T] \times \boldsymbol{R}^{d} \times \boldsymbol{R}$ and the initial function $v$ is bounded and measurable. We deal with this initial value problem (6.1)-(6.2) in the Fréchet space $L_{l o c}^{1}\left(\boldsymbol{R}^{d}\right)$; hence the limit in (6.2) is taken in the sense of the metric topology of $L_{l o c}^{1}\left(\boldsymbol{R}^{d}\right)$.

In what follows, we employ the function space $L_{l o c}^{1}\left(\boldsymbol{R}^{d}\right), L^{\infty}\left(\boldsymbol{R}^{d}\right)$ and $L^{1}\left(\boldsymbol{R}^{d}\right)$. For $v \in L^{\infty}\left(\boldsymbol{R}^{d}\right)$ the $L^{\infty}$-norm of $v$ is denoted by $\|v\|_{\infty}$; and for $v \in L^{1}\left(\boldsymbol{R}^{d}\right)$ the $L^{1}$ norm of $v$ is denoted by $\|v\|_{1}$. Moreover, in order to clarify the relation of the above-mentioned problem to the abstract theory for the evolution equations $\left\{(D E)_{s}\right\}$, we write $X$ and $Y$ for the spaces $L_{l o c}^{1}\left(\boldsymbol{R}^{d}\right)$ and $L^{1}\left(\boldsymbol{R}^{d}\right)$, respectively.

For each $\alpha \geqq 0$, let $G_{\alpha}=[0, T] \times \boldsymbol{R}^{d} \times[-\alpha, \alpha]$. We then put three conditions on the functions $\phi_{i}(t, x, \xi), i=1, \cdots, d$, and $\psi(t, x, \xi)$ :

( I ) The functions $\phi_{i}, \psi$ and their partial derivatives $\phi_{i x_{j}}, \phi_{i \xi}, \phi_{i x_{j} x_{k}}$, $\phi_{i x_{j} \xi}, \psi_{x_{j}}$ and $\psi_{\xi}(i, j, k=1, \cdots, d)$ are continuous in $t, x, \xi$ and are bounded on each $G_{\alpha}$.

(II) There are constants $c \geqq 0$ and $\omega \geqq 0$ such that

$$
-\sum_{i=1}^{d} \phi_{i x_{i} \xi}(t, x, \xi)-\psi_{\xi}(t, x, \xi) \leqq c \quad \text { and }-\psi_{\xi}(t, x, \xi) \leqq \omega
$$

for $(t, x, \xi) \in[0, T] \times \boldsymbol{R}^{d} \times \boldsymbol{R}$.

(III) For each $\alpha \geqq 0$ and $r \in[0, T]$, we denote by $\rho(r ; \alpha)$ the supremum with respect to $x \in \boldsymbol{R}^{d},|\xi| \leqq \alpha,|s-t| \leqq r$ of the maximum of the values

$$
\left|\phi_{i}(t, x, \xi)-\phi_{i}(s, x, \xi)\right|, \quad\left|\phi_{i x_{i}}(t, x, \xi)-\phi_{i x_{i}}(s, x, \xi)\right|, \quad\left|\phi_{i \xi}(t, x, \xi)-\phi_{i \xi}(s, x, \xi)\right|,
$$

and

Then

$$
|\psi(t, x, \xi)-\psi(s, x, \xi)|
$$

$$
\lim _{r \downarrow 0} \rho(r ; \alpha)=0 \quad \text { for each } \quad \alpha \geqq 0 .
$$

In general, the above initial value problem fails to have classical global solutions even if the initial function $v$ is smooth. Hence we mainly consider initial data which belong to $L^{\infty}\left(\boldsymbol{R}^{d}\right)$ and employ the generalized solutions in the sense of Kružkov [9]. 
Given a $v \in L^{\infty}\left(\boldsymbol{R}^{d}\right)$, a real-valued function $u$ on $[0, T] \times \boldsymbol{R}^{d}$ is called a generalized solution of (6.1) with initial value $v$ if it satisfies the following conditions:

$\left(\mathrm{g}_{1}\right) \quad$ For $t \in[0, T], u(t, \cdot) \in L^{\infty}\left(\boldsymbol{R}^{d}\right)$; and there exist constants $c, c^{\prime} \geqq 0$ such that $\|u(t, \cdot)\|_{\infty} \leqq e^{c t}\left(\|v\|_{\infty}+c^{\prime} t\right)$ for $t \in[0, T]$.

$\left(\mathrm{g}_{2}\right) \quad$ For $k \in R$ and $f \in C_{0}^{\infty}\left((0, T) \times \boldsymbol{R}^{d}\right)^{+}$, one has

$$
\begin{gathered}
\int_{0}^{T} \int_{R^{d}}\left(|u(t, x)-k| f_{t}(t, x)+\operatorname{sgn}_{0}(u(t, x)-k) \sum_{i=1}^{d}\left[\phi_{i}(t, x, u(t, x))-\phi_{i}(t, x, k)\right] f_{x_{i}}(t, x)\right. \\
\left.-\operatorname{sgn}_{0}(u(t, x)-k)\left[\psi(t, x, u(t, x))+\sum_{i=1}^{d} \phi_{x_{i}}(t, x, k)\right] f(t, x)\right) d x d t \geqq 0,
\end{gathered}
$$

where $\operatorname{sgn}_{0}(\xi)=1$ if $\xi>0,=0$ if $\xi=0$, and $=-1$ if $\xi<0$.

$\left(\mathrm{g}_{3}\right) \quad$ The $L^{\infty}\left(\boldsymbol{R}^{d}\right)$-valued function $u(t) \equiv u(t, \cdot)$ is continuous over $[0, T]$ in the metric topology of the Fréchet space $X$ and $u(0)=v$.

Now for $v \in X$, set $\mathfrak{p}(v)=\|v\|_{\infty}$ if $v \in L^{\infty}\left(\boldsymbol{R}^{d}\right)$ and $\mathfrak{p}(v)=+\infty$ otherwise. Then the functional $\mathfrak{p}$ defines an l.s.c. seminorm on $X$ with effective domain $D(\mathfrak{p})=$ $L^{\infty}\left(\boldsymbol{R}^{d}\right)$. Hence one can define a family of subsets $\left\{X_{\alpha}: \alpha>0\right\}$ and a set $X_{0}$ by (1.2), i.e.

$$
X_{\alpha}=\left\{v \in L^{\infty}\left(\boldsymbol{R}^{d}\right):\|v\|_{\infty} \leqq \alpha\right\}, \alpha>0, \quad \text { and } \quad X_{0}=L^{\infty}\left(\boldsymbol{R}^{d}\right)
$$

It turns out that initial data under consideration are classified in terms of the $L^{\infty}$-norm. We next construct a family $\left\{P_{\alpha}: \alpha>0\right\}$ of injective linear operators from $X_{0}$ into $Y$, thereby embedding each $X_{\alpha}$ into the Banach space $Y$. Choose an $\varepsilon_{0} \in(0,1)$ and, for each $\alpha>0$, let $K_{\alpha}^{\prime \prime}$ be the maximum of suprema of $\left|\phi_{i}\right|,\left|\phi_{i x_{i}}\right|,\left|\phi_{i \xi}\right|,\left|\phi_{i x_{i} x_{k}}\right|,\left|\phi_{i x_{j} \xi}\right|,|\psi|,\left|\psi_{x_{i}}\right|$ and $\left|\psi_{\xi}\right|$ over $G_{\alpha}$. We then select a monotone nonincreasing function $\alpha \rightarrow \delta_{\alpha}$ from $[0, \infty)$ into $R$ such that $0<\delta_{\alpha} \leqq$ $\min \left\{1,\left(1-\varepsilon_{0}\right) / 2 d K_{\alpha}^{\prime \prime}\right\}$ for $\alpha \geqq 0$, and define two functions $\alpha \rightarrow K_{\alpha}$ and $\alpha \rightarrow h_{\alpha}$ from $[0, \infty)$ into itself by

$$
K_{\alpha}=K_{\alpha}^{\prime} / \delta_{\alpha} \text { and } h_{\alpha}=\min \left\{\varepsilon_{0} / 2 d K_{\alpha}, \varepsilon_{0} / 2, \delta_{\alpha}\right\} \quad \text { for } \quad \alpha \geqq 0,
$$

where $K_{\alpha}^{\prime}$ is $K_{\alpha}^{\prime \prime}$ times the total number of functions $\phi_{i}, \psi$ and their partial derivatives as mentioned above. Notice that the function $\alpha \rightarrow K_{\alpha}$ is nondecreasing while $\alpha \rightarrow h_{\alpha}$ is nonincreasing, and that $K_{\alpha} h_{\alpha} \leqq \varepsilon_{0} / 2$ for $\alpha>0$. Using these functions we define weight functions $p_{\alpha}: \boldsymbol{R}^{d} \rightarrow \boldsymbol{R}, \alpha>0$, by the equation

$$
p_{\alpha}(x)=\left(\rho * \exp \left(-h_{\alpha} \delta_{\alpha}|\cdot|\right)\right)(x)=\int_{R^{d}} \rho(x-y) \exp \left(-h_{\alpha} \delta_{\alpha}|y|\right) d y,
$$

where $\rho$ is any function in $C_{0}^{\infty}\left(\boldsymbol{R}^{d}\right)^{+}$such that $\rho(x)=\rho(|x|)$ for $x \in \boldsymbol{R}^{d}$ and $\int_{R^{d}} \rho(x) d x=1$. Each of the functions $p_{\alpha}$ has the following properties: 


$$
\begin{aligned}
& p_{\alpha}(x)>0 \quad \text { for } \quad x \in \boldsymbol{R}^{d} ;\left\|p_{\alpha}\right\|_{\infty} \leqq 1 ;\left\|p_{\alpha}\right\|_{1} \leqq\left(2 / h_{\alpha} \delta_{\alpha}\right)^{d}, \\
& \left|\left(\partial / \partial x_{j}\right) p_{\alpha}(x)\right| \leqq h_{\alpha} \delta_{\alpha} p_{\alpha}(x) \quad \text { for } x \in \boldsymbol{R}^{d} ; \text { and } \\
& p_{\alpha}(x+y) \leqq \exp \left(h_{\alpha} \delta_{\alpha}|y|\right) p_{\alpha}(x) \quad \text { for } x, y \in \boldsymbol{R}^{d} .
\end{aligned}
$$

Making use of these functions, one can define a family of linear operators $\left\{P_{\alpha}: \alpha>0\right\}$ from $X_{0}$ into $Y$ by

$$
\left[P_{\alpha} v\right](x)=p_{\alpha}(x) v(x) \quad \text { for } \quad x \in \boldsymbol{R}^{d}, v \in X_{0} \quad \text { and } \quad \alpha>0 .
$$

From (6.4) it is seen that $\left\|P_{\alpha} v\right\|_{\infty} \leqq\|v\|_{\infty},\left\|P_{\alpha} v\right\|_{1} \leqq\left(2 / h_{\alpha} \delta_{\alpha}\right)^{d}\|v\|_{\infty}$ for $v \in X_{0}$ and $\alpha>0$, and that $\left\|P_{\alpha} v\right\|_{1}>0$ for $v \in X_{0}-\{0\}$. Hence each $P_{\alpha}$ maps $X_{0}$ into $X_{0} \cap Y$ and is injective over $X_{0}$; and each of the absolutely convex closed subsets $X_{\alpha}, \alpha>0$, is embedded into the Banach space $Y=L^{1}\left(\boldsymbol{R}^{d}\right)$ in the sense of condition (F). According to (2.1), we write $\|v\|_{\alpha}=\left\|P_{\alpha} v\right\|_{1}$ for $v \in X_{0}$ in the following.

We now find a one-parameter family $\{A(t): t \in[0, T]\}$ of operators in $X$ satisfying conditions $\left(\mathbf{A}^{\prime} . \mathbf{i}\right)$ and $\left(\mathbf{S}^{\prime} . \mathbf{i}\right), i=1,2,3$. First, referring to Crandall [4] and Oharu [10], we define operators $A(t), t \in[0, T]$, by the following: $(v, w)$ $\in A(t)$ iff $(v, w) \in X_{0} \times X_{0}$ and for $k \in R$ and $f \in C_{0}^{\infty}\left(\boldsymbol{R}^{d}\right)^{+}$we have

$$
\begin{aligned}
& \int_{R^{d}} \operatorname{sgn}_{0}(v(x)-k)\left[\sum_{i=1}^{d}\left(\phi_{i}(t, x, v)-\phi_{i}(t, x, k)\right) f_{x_{i}}(x)\right. \\
& \left.\quad-\left(\sum_{i=1}^{d} \phi_{i x_{i}}(t, x, k)+\psi(t, x, v)\right) f(x)-w(x) f(x)\right] d x \geqq 0 .
\end{aligned}
$$

Taking $k=\|v\|_{\infty}+1$ and then $k=-\|v\|_{\infty}-1$, we infer that $-\sum_{i=1}^{d}\left(\phi_{i}(t, x, v)\right)_{x_{i}}=$ $\psi(t, x, v)+w$ in $\mathscr{D}^{\prime}\left(\boldsymbol{R}^{i}\right)$. Hence each $A(t)$ is a singlevalued operator with domain $D(A(t))$ and range $R(A(t))$ contained in $X_{0}$. There are nontrivial examples which show that $D(A(t))$ is in general a proper subset of the maximal domain of the differential operator in $X_{0}$

$$
v \rightarrow \sum_{i=1}^{d}\left(\phi_{i}(t, x, v)\right)_{x_{i}}, \quad(t, x) \in[0, T] \times \boldsymbol{R}^{d},
$$

and that $D(A(t))$ contains properly the space $W_{1}^{\infty}\left(\boldsymbol{R}^{d}\right)$. See [4]. If in particular $d=1$, then $-\left(\phi_{1}(t, x, u)\right)_{x}=\psi(t, x, u)+w$ in $\mathscr{D}^{\prime}(\boldsymbol{R})$. Since the right side lies in $L^{\infty}(\boldsymbol{R})$, the Radon-Nikodym theorem implies that $\phi(t, \cdot, v) \in W_{1}^{\infty}(\boldsymbol{R})$ and $w=$ $-(\phi(t, \cdot, v))_{x}-\psi(t, \cdot, v)$ in $L^{\infty}(\boldsymbol{R})$.

Accordingly, the initial value problem (6.1)-(6.2) can be written as the evolution equation in $X$ :

$$
u^{\prime}(t)=A(t) u(t), \quad 0<t<T ; \quad u(0)=v \in X_{0} .
$$

We then show that the family of operators $\{A(t): t \in[0, T]\}$ defined above satisfies conditions treated in the preceding sections.

Firstly, to show that $\{A(t)\}$ satisfies $\left(\mathbf{A}^{\prime} \cdot \mathbf{1}\right)$, it suffices to verify that $D(A(t))$ 
$\cap X_{\beta}$ is dense in $X_{\beta}$ for $\beta>0$. To this end let $\rho_{n} *$ be the usual Friedrichs mollifier with support $[-1 / n, 1 / n]$ for every positive integer $n$. Let $v \in X_{\beta}$ and let $v_{n}=\rho_{n} * v=\int_{\boldsymbol{R}^{d}} \rho_{n}(\cdot-y) v(y) d y$ for $n \geqq 1$. Then $v_{n} \in W_{1}^{\infty}\left(\boldsymbol{R}^{d}\right),\left\|v_{n}\right\|_{\infty} \leqq\|v\|_{\infty} \leqq \beta$ for $n \geqq 1$, and $v_{n} \rightarrow v$ in $X$ as $n \rightarrow \infty$. Since $W_{1}^{\infty}\left(\boldsymbol{R}^{d}\right) \subset D(A(t))$ (see [5] and [6]), we infer that $v$ lies in the closure of $D(A(t)) \cap X_{\beta}$. Thus $D(A(t)) \cap X_{\beta}$ is dense in $X_{\beta}$.

Secondly, it was shown in the recent work of Yanagihara and Oharu [6] that the inequality

$$
\|(I-\lambda A(t)) v-(I-\lambda A(t)) w\|_{\alpha} \geqq\left[1-\lambda\left(\omega+\varepsilon_{0}\right)\right]\|v-w\|_{\alpha}
$$

holds for $v, w \in D(A(t)) \cap X_{\alpha}, \lambda \in\left(0,1 /\left(\omega+\varepsilon_{0}\right)\right)$ and $\alpha>0$. We note that in the verification of (6.7) Kruzkov's method developed in [9] is employed and the properties listed in (6.4) of the functions $p_{\alpha}$ are fully utilized. The inequality (6.7) states that the operator $A(t)-\left(\omega+\varepsilon_{0}\right)$ is dissipative on $X_{\alpha}$ with respect to the norm $\|\cdot\|_{a}$ for each $\alpha>0$. Therefore, if $\left(\omega+\varepsilon_{0}\right) \lambda<1$ then $I-\lambda A(t)$ is injective and the resolvent $J_{\lambda}(t) \equiv(I-\lambda A(t))^{-1}$ exists as a single-valued operator in $X$. In what follows, we set

$$
\lambda_{0}=\min \left\{\left(\omega+\varepsilon_{0}\right)^{-1}\left(1-\varepsilon_{0}^{2}\right),\left(c+\varepsilon_{0}\right)^{-1}\left(1-\varepsilon_{0}^{2}\right)\right\} .
$$

Thirdly, we define for each $\alpha>0$ a functional $\mathfrak{q}_{\alpha}: X \rightarrow[0, \infty]$ by

$$
\mathfrak{q}_{\alpha}(w)=\sup \left\{|y|_{1}^{-1}\left\|\prod_{i=1}^{d} \tau_{i y_{i}} w-w\right\|_{\alpha}: 0<|y|_{1} \leqq 1\right\}
$$

for $w \in X_{0}$; and $q_{\alpha}(w)=+\infty$ otherwise, where $y=\left(y_{1}, \cdots, y_{d}\right),|y|_{1}=\sum_{i=1}^{d}\left|y_{i}\right|$ and $\tau_{i y_{i}}$ denotes the translation operator defined by $\left[\tau_{i y_{i}} w\right](x)=w\left(x+y_{i} e_{i}\right)$, $e_{i}=(0, \cdots, \stackrel{(i)}{1}, \cdots, 0)$. It is easy to see that each $\mathfrak{q}_{\alpha}$ is l.s.c. over $X$ and specifies when functions in $X_{0}$ are locally of bounded variation over $\boldsymbol{R}^{d}$. Further, the first half of condition $\left(\mathbf{S}^{\prime} .2\right)$ is verified with the functionals $\mathfrak{q}_{\alpha}, \alpha>0$. In fact, let $\alpha, \beta>0, v \in X_{\beta}$, and let $v_{n}=\rho_{n} * v, n=1,2,3, \cdots$, as before. Then we have $|y|_{1}^{-1} \int_{R^{d}} p_{\alpha}(x)\left|v_{n}(x+y)-v_{n}(x)\right| d x \leqq\left\|v_{n}^{\prime}\right\|_{\infty}\left\|p_{\alpha}\right\|_{1}$ for $y \neq 0$ and $n \geqq 1$. This means that $v_{n} \in D\left(\mathfrak{q}_{\alpha}\right) \cap X_{\beta}$ for $n \geqq 1$, and so $D\left(\mathfrak{q}_{\alpha}\right) \cap X_{\beta}$ is dense in $X_{\beta}$.

We use the same notation as in Section 5: Given an $\alpha>0, A_{\alpha}(t)$ denotes the restriction of $A(t)$ to the set $X_{\alpha}$ and $J_{\alpha}(\lambda ; t)$ the resolvent $\left(I-\lambda A_{\alpha}(t)\right)^{-1}$ wherever it makes sense. We now state a result due to Oharu and Yanagihara [6].

Proposition 6.1. Let $t \in[0, T]$ and $\beta>0$. Choose an $\alpha>0$ with $\alpha \geqq\left(1-c \lambda_{0}\right)^{-1}\left[\beta+\left(c^{\prime}+\varepsilon_{0}\right) \lambda_{0}\right], c^{\prime}=\sup _{G_{0}}\left(\left|\sum_{i=1}^{d} \phi_{i x_{i}}\right|+|\psi|\right)$, and define

$$
\lambda_{\alpha}=\min \left\{\lambda_{0},\left(d k_{\alpha}^{\prime \prime}+\varepsilon_{0}\right)^{-1}\left(1-\varepsilon_{0}^{2}\right)\right\} .
$$


Then the operator $A(t)$ has the following properties:

$\left(1^{\circ}\right) \quad R\left(I-\lambda A_{\alpha}(t)\right) \supset X_{\beta}$ for $\lambda \in\left(0, \lambda_{\alpha}\right)$ and $D\left(A_{\alpha}(t)\right) \supset W_{1}^{\infty}\left(\boldsymbol{R}^{d}\right) \cap X_{\alpha}$.

$\left(2^{\circ}\right) \quad\left\|J_{\alpha}(\lambda ; t) v-J_{\alpha}(\lambda ; t) w\right\|_{\alpha} \leqq\left(1-\lambda\left(\omega+\varepsilon_{0}\right)\right)^{-1}\|v-w\|_{\alpha}$ for $v, w \in X_{\beta}$ and $\lambda \in$ $\left(0, \lambda_{\alpha}\right)$.

$\left(3^{\circ}\right) \quad\left\|J_{\alpha}(\lambda ; t) v\right\|_{\infty} \leqq(1-c \lambda)^{-1}\left(\|v\|_{\infty}+c^{\prime} \lambda\right)$ for $v \in X_{\beta}$ and $\lambda \in\left(0, \lambda_{\alpha}\right)$.

$\left(4^{\circ}\right) \quad$ There exists a constant $L_{\alpha} \geqq 0$ such that

$$
\mathfrak{q}_{\alpha}\left(J_{\alpha}(\lambda ; t) v\right) \leqq \exp \left(L_{\alpha} \lambda\right)\left[q_{\alpha}(v)+L_{\alpha} \lambda\right]
$$

for $v \in X_{\beta}$ with $\mathfrak{q}_{\alpha}(v)<\infty$ and $\lambda \in\left(0, \lambda_{\alpha}\right)$.

$\left(5^{\circ}\right)$ Let $\delta>0$ and $\gamma \geqq \exp \left(L_{\alpha} \lambda_{\alpha}\right)\left(\delta+L_{\alpha} \lambda_{\alpha}\right)$. Then there exists a constant $L_{\alpha}^{r} \geqq 0$ such that

$$
\left\|J_{\alpha}(\lambda ; s) v-J_{\alpha}(\lambda ; t) v\right\|_{\alpha} \leqq \lambda L_{\alpha}^{\gamma} \rho(|s-t| ; \alpha)
$$

for $v \in X_{\beta}$ with $\mathfrak{q}_{\alpha}(v) \leqq \delta$ and $\lambda \in\left(0, \lambda_{\alpha}\right)$, where $\rho(\cdot ; \alpha)$ is the function specified as in Assumption (III).

From Proposition 6.1 above and (6.7) it follows that the family $\{A(t)\}$ satisfies conditions (A.2), ( $\left.\mathbf{A}^{\prime} .3\right),\left(\mathbf{S}^{\prime} .1\right)$, and the last half of $\left(\mathbf{S}^{\prime} .2\right)$. Moreover, we infer from the fact $\left(5^{\circ}\right)$ that $\{A(t)\}$ satisfies $\left(\mathbf{S}^{\prime} .3\right)$ in the following way: Set $\theta_{\alpha}^{\gamma}(t, s)=L_{\alpha}^{\gamma} \rho(|t-s| ; \alpha)$ for $s, t \in[0, T]$ in $\left(5^{\circ}\right)$. Then Assumption (III) implies that $\theta_{\alpha}^{\tau}$ belongs to the calss $\mathscr{C}$, and $\{A(t)\}$ satisfies condition $\left(\mathbf{S}^{\prime} .3\right)$. In what follows, set $f(\beta)=\left(1-c \lambda_{0}\right)^{-1}\left[\beta+\left(c^{\prime}+\varepsilon_{0}\right) \lambda_{0}\right]$ for $\beta>0$.

Thus, Theorem 2 can be applied to obtain an evolution operator $\mathscr{U}=$ $\{U(t, s): 0 \leqq s \leqq t \leqq T\}$ on $X_{0}$ with the following properties:

(a)" For $s \in[0, T), t \in[s, T]$ and $v \in X_{0}$, one has the convergence

$$
U(t, s) v=L_{l o c}^{1}-\lim _{\lambda \downarrow 0} \prod_{p=1}^{[(t-s) / \lambda]}(I-\lambda A(s+p \lambda))^{-1} v .
$$

(b)" For $\beta>0, \beta^{\prime} \geqq e^{2 c T}\left(\beta+c^{\prime} T\right), \alpha \geqq f\left(\beta^{\prime}\right)$, and $v \in X_{\beta}$, the $X_{0}$-valued function $u(t)=U(t, 0) v$ gives a unique weak solution with initial-value $v$ of (6.6) constrained in $X_{\alpha}$. Moreover, the relation of $U$ to the equation (6.1) is stated as follows: For every $v \in X_{0}$, the real-valued function $u(t, x) \equiv[U(t, 0) v](x)$ on $[0, T] \times \boldsymbol{R}^{d}$ is a unique generalized solution of (6.1)-(6.2) in the sense of Kružkov.

(c) $)^{\prime \prime}$ For $\beta>0, \beta^{\prime} \geqq e^{2 c T}\left(\beta+c^{\prime} T\right), \alpha \geqq f\left(\beta^{\prime}\right), v, w \in X_{\beta}$, and $s, t \in[0, T]$ with $s \leqq t$, we have

$$
\|U(t, s) v-U(t, s) w\|_{\alpha} \leqq \exp \left[\left(\omega+\varepsilon_{0}\right)(t-s)\right]\|v-w\|_{\alpha} .
$$

$(\mathrm{d})^{\prime \prime} \quad\|U(t, s) v\|_{\infty} \leqq e^{c(t-s)}\left[\|v\|_{\infty}+c^{\prime}(t-s)\right]$ for $v \in X_{0}$ and $0 \leqq s \leqq t \leqq T$.

(e)" Let $\beta>0, \delta>0, \beta^{\prime} \geqq e^{2 c T}\left(\beta+c^{\prime} T\right), \alpha>f\left(\beta^{\prime}\right)$, and let $\gamma>e^{L_{\alpha} T}\left(\delta+L_{\alpha} T\right)$.

Then the inequality $\mathfrak{q}_{\alpha}(U(t, s) v) \leqq \exp \left[L_{\alpha}(t-s)\right]\left[\mathfrak{q}_{\alpha}(v)+L_{\alpha}(t-s)\right] \leqq \gamma$ holds for $0 \leqq s \leqq t \leqq T$ and $v \in X_{\beta}$ with $\mathfrak{q}_{\alpha}(v) \leqq \delta$. 
In case the space dimension is one, the family $\{A(t)\}$ has more precise properties than those listed in $\left(1^{\circ}\right)$ through $\left(5^{\circ}\right)$. In fact, it is proved in Kobayasi and Oharu [5], Section, 2, that if $d=1$ then the associated family of operators $\{A(t)\}$ has the properties listed below.

Proposition 6.2. Let $d=1 . \quad$ Let $\beta>0, \lambda \in\left(0, \lambda_{0}\right), s, t \in[0, T]$, and let $\alpha \geqq$ $(1-c \lambda)^{-1}\left[\beta+\left(c^{\prime}+\varepsilon_{0}\right) \lambda\right]$. Then we have:

$\left(1^{\circ}\right)^{\prime} \quad R(I-\lambda A(t))=L^{\infty}(R) \supset D(A(t)) \supset W_{1}^{\infty}(R) ;$

$\left(2^{\circ}\right)^{\prime} \quad\|J(\lambda ; t) v-J(\lambda ; t) w\|_{\alpha} \leqq\left[1-\left(\omega+h_{\alpha}\right) \lambda\right]^{-1}\|v-w\|_{\alpha}$ for $v, w \in X_{\beta}$;

$\left(3^{\circ}\right)^{\prime} \mathfrak{p}(J(\lambda ; t) v) \leqq(1-c \lambda)^{-1}\left[\mathfrak{p}(v)+c^{\prime} \lambda\right]<\alpha$ for $v \in X_{\beta} ;$ and

$\left(4^{\circ}\right)^{\prime} \quad \mathfrak{q}_{\alpha}(J(\lambda ; t) v) \leqq \exp \left(L_{\alpha} \lambda\right)\left[\mathfrak{q}_{\alpha}(v)+L_{\alpha} \lambda\right]$ for $v \in X_{\beta}$,

where $L_{\alpha}$ is a constant depending only on $\alpha$ and $\mathfrak{q}_{\alpha}$ is an l.s.c. seminorm on $X$ denfied by $\mathfrak{q}_{\alpha}(v)=\sup \left\{|y|^{-1}\|v(\cdot+y)-v(\cdot)\|_{\alpha}: y \neq 0,|y| \leqq 1\right\}$.

$\left(5^{\circ}\right)^{\prime} \quad$ Let $\beta>0, \delta>0, \lambda \in\left(0, \lambda_{0}\right), s, t \in[0, T], \alpha>(1-c \lambda)^{-1}\left[\beta+\lambda\left(c^{\prime}+\varepsilon_{0}\right)\right]$, and let $\gamma>\exp \left(L_{\alpha} \lambda\right)\left[\delta+L_{\alpha} \lambda\right], L_{\alpha}$ being the constant as stated in $\left(4^{\circ}\right)$. Let $\rho(\cdot ; \alpha)$ : $[0, T] \rightarrow \boldsymbol{R}$ be the function specified as in Assumption (III). Then there exists $a$ number $L_{\alpha}^{r}>0$, depending only upon $\alpha$ and $\gamma$, such that

$$
\|J(\lambda ; t) v-J(\lambda ; s) v\|_{\alpha} \leqq \lambda L_{\alpha}^{\gamma} \rho(|t-s| ; \alpha) \quad \text { for } \quad v \in X_{\beta} \text { with } \mathfrak{q}_{\alpha}(v) \leqq \delta .
$$

Therefore it follows that $\{A(t)\}$ satisfies conditions (F), (A.1) and (S.i), $i=1,2,3$, which are stated in Section 1 , and Theorem 1 is directly applied to get the conclusions $(a)^{\prime \prime}-(e)^{\prime \prime}$ as mentioned above. Moreover a little more precise relation of the evolution operator $\mathscr{U}$ to the equation (6.1) with $d=1$ can be obtained: For every $v \in L^{\infty}(\boldsymbol{R})$, the $L^{\infty}(\boldsymbol{R})$-valued function $u(t) \equiv U(t, 0) v$ satisfies the integeral equation

$$
u(t)-v+\Lambda \int_{0}^{t} \Phi(s) u(s) d s+\int_{0}^{t} \Psi(s) u(s) d s=0 \quad \text { for } \quad t \in[0, T]
$$

where $A: W_{1}^{\infty}(R) \rightarrow L^{\infty}(R)$ is the differential operator defined by $\Lambda v=v^{\prime}$ for $v \in W_{1}^{\infty}(\boldsymbol{R}) ; \Phi(t): L^{\infty}(\boldsymbol{R}) \rightarrow L^{\infty}(\boldsymbol{R}), \Psi(t): L^{\infty}(\boldsymbol{R}) \rightarrow L^{\infty}(\boldsymbol{R})$ are composition operators defined respectively by

$$
[\Phi(t) v](x)=\phi(t, x, v(x)),[\Psi(t) v](x)=\psi(t, x, v(x)) \quad \text { for } \quad v \in L^{\infty}(R) ;
$$

and the integrals are taken in $X$ in the sense of Riemann. The relation (6.8) implies that the $L^{\infty}(R)$-valued function $u(t)$ satisfies the equation (that is a generalized form of (6.1)):

$$
(d / d t)\langle u(t), f\rangle=\left\langle\Phi(t) u(t), f^{\prime}\right\rangle+\langle-\Psi(t) u(t), f\rangle
$$

for $0<t<T$ and $f \in C_{0}^{1}(R)$, where $\langle v, f\rangle$ denotes the integral $\int_{R} v(s) f(s) d s$ for $v \in L_{l o c}^{1}(R)$ and $f \in C_{0}^{1}(R)$. 


\title{
References
}

[1] M. Crandall, A generalized domain for semigroup generators, Proc. Amer. Math. Soc., 37 (1973) , 434-440.

[2] M. Crandall and T. Liggett, Generation of semigroups of nonlinear transformations on general Banach spaces, Amer. J. Math., 93 (1971), 265-293.

[ 3 ] M. Crandall and A. Pazy, Nonlinear evolution equations in Banach spaces, Israel J. Math., 11 (1972), 57-94.

[4] M. Crandall, The semigroup approach to first order quasilinear equations in several space variables, Israel J. Math., 12 (1972), 108-132.

[5] K. Kobayasi and S. Oharu, On nonlinear evolution operators associated with certain nonlinear equations of evolution, Lecture Notes in Appl. Anal,, 2, Kinokuniya Book Store Co., Tokyo, 1980, 139-210.

[6] S. Oharu and A. Yanagihara, On the finite-difference approximation of a certain nonlinear operator in the space $L_{l o c}^{1}\left(\boldsymbol{R}^{d}\right)$, to appear.

[ 7 ] K. Kobayasi, Y. Kobayashi and S. Oharu, Nonlinear evolution operators in Banach spaces, Osaka Math. J., 21 (1984), 281-310.

[ 8 ] Y. Kobayashi, Difference approximation of Cauchy problems for quasidissipative operators and generation of nonlinear semigroups, J. Math. Soc. Japan, 27 (1975), 640-665.

[9] S. Kružkov, First order quasilinear equations in several independent variables, Math. USSR-Sb., 10 (1970), 217-243.

[10] S. Oharu, Nonlinear semigroups and finite-difference method, Functional Analysis and Numerical Analysis, Japan-France Seminar, Tokyo and Kyoto, 1976; H. Fujita (Ed.): Japan Society for the Promotion of Science, 1978, 383-402.

\author{
Kazuo KoBAyASI \\ Department of MATHematics \\ Sagami Institute of Technology \\ Fujisawa, Kanagawa 251 \\ Shinnosuke OHARU \\ DEPARTMENT OF MATHEMATICS \\ Faculty of Science \\ Hiroshima University \\ HIROSHIMA 730
}

